\title{
Approximate controllability of nonlinear stochastic partial differential systems with infinite delay
}

\author{
Hanwen Ning ${ }^{1 *}$ and Guangyan Qing ${ }^{2}$
}

\author{
"Correspondence: \\ ninghanwen@gmail.com \\ ${ }^{1}$ School of Statistics and \\ Mathematics, Zhongnan University \\ of Economics and Law, Wuhan, \\ 430073, P.R. China \\ Full list of author information is \\ available at the end of the article
}

\begin{abstract}
This paper aims to investigate approximate controllability of stochastic nonlinear partial differential systems with infinite delay. In the systems under study, nonlinearity and control variable exist both in drift and diffusion terms, and controllability problems are considered in the framework with a novel Banach space, which not only leads to some difficulties in deriving the properties of interest but also bring some opportunities to study the system in a more general framework. With the help of a new fundamental lemma established in this paper and some useful inequality techniques, some improved results for approximate controllability of stochastic partial differential systems are obtained by using the Banach contraction theorem without introducing any additional restraints on the terms of the system. An example of stochastic heat equation is also provided to illustrate our results.
\end{abstract}

Keywords: stochastic; partial differential systems; approximate controllability; Banach contraction theorem; infinite delay

\section{Introduction}

Stochastic partial differential systems are usually used to describe physical and engineering phenomena such as heat process, population dynamics, chemical reactors, fluid dynamics, etc. and have been widely investigated (for instance, [1-10] and references therein). As an important concept in control theory, controllability of dynamical systems has been also investigated by many researchers. In [11-14], Mahmudov developed several concepts of controllability for linear stochastic differential systems and extended the classical theory for deterministic dynamical systems to stochastic cases. The authors in [15, 16] considered weak, complete, and exact controllability of semilinear stochastic systems and stochastic functional differential in Hilbert spaces, and the obtained results therein can be applied to the stochastic systems with kinds of delays. Moreover, with the help of semigroup theory, sufficient conditions for the controllability of stochastic integrodifferential systems were derived in $[17,18]$.

It should be also noted that the controllability problems can be transformed into fixed point problems. Fixed point principles such as Banach contraction theorem, Nussbaum theorem and Schauder fixed point theorem are frequently used and considered as an important technique in solving the controllability problems. By employing an axiomatic definition of the phase space introduced in [19, 20], Balasubramaniam and Ntouyas [21] inves- 
tigated controllability of neutral stochastic differential inclusions with infinite delay with the aid of Leray-Schauder nonlinear alternative. Sufficient conditions for controllability of neutral functional integrodifferential infinite delay systems in Banach spaces were studied in [22] with the Nussbaum fixed point theorem. Bao and Jiang [23] considered the approximate controllability of stochastic partial differential equations with infinite delay. Recently, in [24, 25], by using the Schauder fixed point theorem and the fixed point theorem for condensing maps due to Martelli, the authors derived some sufficient conditions for controllability of functional differential systems with infinite delay in a deterministic case.

However, most of the results for the stochastic systems mentioned above focus on either finite delays or without delays. Since many systems arising from realistic models greatly depend on histories, it is highly relevant to discuss stochastic differential systems with infinite delays, and few works are available for the controllability properties on this case. Therefore, in this paper, we study the approximate controllability of a class of nonlinear stochastic partial differential systems with infinite delays. This kind of system can be found in many engineering practices, especially those relating to continuum physics, vibration control, and thermodynamics [26-31]. Nonlinearity and control input exist both in the drift and diffusion terms of the underlying equation, and the control problem of interest is considered in the framework with a novel Banach space. This may not only enable the system under study to be a more general case compared to [23], but also bring some difficulties in moment estimations and in employing the fixed point theorem. To this objective, an important lemma is established, which greatly facilitates the development of the main result of this paper. Together with the aid of some useful inequality formula adopted in the proof, improved approximate controllability results of the systems under study can be obtained without imposing any additional restraints on the system. An example is given to illustrate our results.

The rest of the paper is organized as follows. In Section 2, we introduce the basic notations and definitions. In Section 3.1, we introduce Banach spaces $B_{h}$ and $B_{h}^{\alpha}$ and prove an important inequality. In Section 3.2, we give the controllability results. In the last section, an example of stochastic heat equation is given to illustrate our results.

\section{Preliminaries}

In this section, we will briefly give some basic assumptions and definitions.

Let $K$ and $H$ be two separable Hilbert spaces. We denote by $|\cdot|$ and $|\cdot|_{K}$ the norms in $H$ and $K$, respectively, by $\langle\cdot, \cdot\rangle$ the scalar product in $H . L(K, H)$ denotes the space of all bounded linear operators from $K$ into $H .\|\cdot\|$ is also used to denote the norm in an ordinary Banach space. Let $\left(\Omega, \Im_{\mathfrak{I}}, \Im_{t}, P\right)$ be a complete probability space with a filtration $\left\{\Im_{t}\right\}$ satisfying the usual condition (i.e., the filtration contains all P-null sets and is right continuous). Let $w_{n}(t)(n=1,2,3, \ldots)$ be a sequence of real-valued one-dimensional standard Brownian motions mutually independent on $\left(\Omega, \mathfrak{\Im}, \mathfrak{\Im}_{t}, P\right)$. Set

$$
W(t)=\sum_{n=1}^{\infty} \sqrt{\sigma_{n}} w_{n}(t) e_{n}, \quad t \geq 0,
$$

where $e_{n}(n=1,2,3, \ldots)$ is a complete orthonormal basis in $K$. Let $Q \in L(K, K)$ be an operator defined by $Q e_{n}=\sigma_{n} e_{n}$ with $\sum_{n=1}^{\infty} \sigma_{n}<\infty$. For a Hilbert-Schmidt operator $G$ in $L(K, H)$, 
we denote by $\|G\|_{2}$ its Hilbert-Schmidt norm, i.e.,

$$
\|G\|_{2}^{2}=\operatorname{tr}\left(G Q G^{*}\right) .
$$

We first give an abstract phase space $B_{h}$.

Assume that $h:(-\infty, 0] \rightarrow(0, \infty)$ is a continuous function with $l=\int_{-\infty}^{0} h(s) d s<\infty$. Define

$$
\begin{aligned}
B_{h}= & \{\phi:(-\infty, 0] \rightarrow H \mid \phi \text { is continuous on }[-a, 0] \text { for any } a>0 \text {, and } \\
& \left.\int_{-\infty}^{0} h(s) \sup _{s \leq \theta \leq 0}|\phi(\theta)| d s<\infty\right\} .
\end{aligned}
$$

$B_{h}$ is a Banach space endowed with the norm

$$
\|\phi\|_{B_{h}}=\int_{-\infty}^{0} h(s) \sup _{s \leq \theta \leq 0}|\phi(\theta)| d s \quad \text { for } \forall \phi \in B_{h} .
$$

The above conclusion will be proved in the next section. In the present paper, we are interested in the controllability problem of the following system:

$$
\left\{\begin{array}{l}
d x(t)=\left[-A x(t)+B u(t)+f\left(t, x_{t}, u(t)\right)\right] d t+g\left(t, x_{t}, u(t)\right) d w(t), \quad t \in[0, T), \\
x(t)=\xi(t), \quad t \in(-\infty, 0]
\end{array}\right.
$$

where $-A$ is a closed, densely defined linear operator generating an analytic semigroup $S(t), t \geq 0$ on $H$. Let $0<\alpha<1$, and define the Banach space $D\left(A^{\alpha}\right)$ with the norm $\|x\|_{\alpha}=\left|A^{\alpha} x\right|$ for $x \in D\left(A^{\alpha}\right)$, where $D\left(A^{\alpha}\right)$ denotes the domain of a fractional power operator $A^{\alpha}: H \rightarrow H$ [4]. Denote $H_{\alpha}=D\left(A^{\alpha}\right)$, define $B_{h}^{\alpha}=\{\phi:(-\infty, 0] \rightarrow$ $H_{\alpha} \mid \phi$ is continuous on $[-a, 0]$ for any $a>0$, and $\left.\int_{-\infty}^{0} h(s) \sup _{s \leq \theta \leq 0}\|\phi(\theta)\|_{\alpha} d s<\infty\right\}$. Let $U$ be another separable Hilbert space, $u(t)$ is a $U$ valued process and $B$ is a bounded linear operator from $U$ to $H$. We also assume that $f:\left(R^{+} \times B_{h}^{\alpha} \times U\right) \rightarrow H$ and $g:\left(R^{+} \times B_{h}^{\alpha} \times U\right) \rightarrow$ $L_{2}^{0}(K, H)$ are two measurable mappings, where $L_{2}^{0}(K, H)$ denotes the space of bounded linear operators from $K$ to $H$ with the Hilbert-Schmidt norm. For initial datum $\xi$, let $\xi \in L^{p}\left(\Omega, \Im, P ; B_{h}^{\alpha}\right) \equiv L^{p}\left(\Omega, B_{h}^{\alpha}\right)$, and we always assume $p>2$. Moreover, denote histories $x_{t}:(-\infty, 0] \rightarrow H$ by $x_{t}(\theta)=x(t+\theta)$ for $-\infty<\theta \leq 0$.

Definition 2.1 A stochastic process $x$ is said to be a mild solution of (1) if the following conditions are satisfied.

(1) $x(t, \omega)$ is measurable as a function from $[0, T] \times \Omega$ to $H$, and $x(t)$ is $\Im_{t}$ adapted.

(2) $E|x(t)|^{p}<\infty$ for each $t \in(-\infty, 0]$.

(3) For each $u \in L_{\Im_{t}}^{1}\left(\Omega ; L^{p}(0, T ; U)\right)\left(u(t)\right.$ is $\Im_{t}$ adapted, and $\left.E \int_{0}^{T}\|u(t)\|^{p} d t<\infty\right)$ the process $x$ satisfies the following equation:

$$
\begin{aligned}
x(t)= & S(t) \xi(0)+\int_{0}^{t} S(t-s) B u(s) d s+\int_{0}^{t} S(t-s) f\left(s, x_{s}, u(s)\right) d s \\
& +\int_{0}^{t} S(t-s) g\left(s, x_{s}, u(s)\right) d w(s), \quad t>0, \\
x_{0}=\xi & \in L^{p}\left(\Omega, B_{h}^{\alpha}\right) .
\end{aligned}
$$


Remark 2.2 The dynamic system is controlled by $u(t)$. Sometimes, control term $u(t)$ affects the system not only in a linear manner, but also in a nonlinear way [32, 33]. Especially for stochastic differential systems, $u(t)$ exists in both the diffusion and drift terms. These cases are all taken into consideration in the present study.

Definition 2.3 System (1) is said to be approximately controllable on $[0, T]$ if

$$
\overline{R(T)}=L^{p}(\Omega, \Im, P ; H),
$$

where $R(T)=\left\{x(T)=x(T, u): u \in L^{p}(0, T ; U)\right\}$.

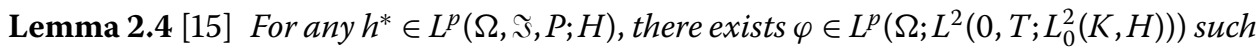
that

$$
h^{*}=E h^{*}+\int_{0}^{T} \varphi(t) d w(t)
$$

Lemma 2.5 [15] If $\varphi \in L^{2}\left(0, T ; L_{0}^{2}(K, H)\right), A^{\alpha} \varphi \in L^{2}\left(0, T ; L_{0}^{2}(K, H)\right)$ and $\varphi(t) k \in H_{\alpha}$ for $t \geq$ 0 and arbitrary $k \in K$, then

$$
A^{\alpha} \int_{0}^{t} \varphi(s) d w(s)=\int_{0}^{t} A^{\alpha} \varphi(s) d w(s) .
$$

Lemma 2.6 Let $p>2, \Sigma \in L^{p}\left(\Omega ; L^{2}\left(0, T ; L_{2}^{0}(K, H)\right)\right)$, we have

$$
\begin{aligned}
E\left(\sup _{0 \leq s \leq t}\left|\int_{0}^{s} \Sigma(r) d w(r)\right|^{p}\right) & \leq c_{p} \sup _{0 \leq s \leq t} E\left|\int_{0}^{s} \Sigma(r) d w(r)\right|^{p} \\
& \leq C_{p} E\left(\int_{0}^{t}\|\Sigma(r)\|_{2}^{2} d r\right)^{\frac{p}{2}}, \quad t \in[0, T],
\end{aligned}
$$

where $c_{p}=\left(\frac{p}{p-1}\right)^{p}$ and $C_{p}=\left(\frac{p}{2}(p-1)\right)^{\frac{p}{2}}\left(\frac{p}{p-1}\right)^{\frac{p^{2}}{2}}$.

Lemma 2.7 [4] Let $-A$ be the infinitesimal generator of an analytic semigroup $S(t)$. If $0 \in$ $\rho(A)$, then

(1) There exist a constant $M \geq 1$ and a real number $a>0$ such that

$$
|S(t) h| \leq M e^{-a t}|h| \quad \text { for all } t \geq 0 \text { and } h \in H .
$$

(2) There exists a constant $M_{\alpha}$ such that the fractional power operator $A^{\alpha}$ satisfies that

$$
\left|A^{\alpha} S(t) h\right| \leq M_{\alpha} e^{-a t} t^{-\alpha}|h| \quad \text { for all } t \geq 0 \text { and } h \in H .
$$

(3) Let $0<\alpha \leq 1$ and $h \in D\left(A^{\alpha}\right)$, there exists a constant $N_{\alpha}$ such that

$$
|S(t) h-h| \leq N_{\alpha} t^{\alpha}\left|A^{\alpha} h\right| \quad \text { for all } t \geq 0 \text { and } h \in H .
$$

For system (1), we have the following hypotheses: 
$\left(\mathrm{A}_{1}\right) \quad 0 \in \rho(A)$.

$\left(\mathrm{A}_{2}\right)$ For any $\eta_{1}, \eta_{2} \in B_{h}^{\alpha}, v_{1}, v_{2} \in U$ and $0 \leq t \leq T$, there exists a constant $K_{1}$ such that

$$
\begin{aligned}
& \left|f\left(t, \eta_{1}, v_{1}\right)-f\left(t, \eta_{2}, v_{2}\right)\right|^{p}+\left\|g\left(t, \eta_{1}, v_{1}\right)-g\left(t, \eta_{2}, v_{2}\right)\right\|_{2}^{p} \\
& \quad \leq K_{1}\left(\left\|\eta_{1}-\eta_{2}\right\|_{B_{h}^{\alpha}}^{p}+\left\|v_{1}-v_{2}\right\|^{p}\right), \\
& \left|f\left(t, \eta_{1}, v_{1}\right)\right|^{p}+\left\|g\left(t, \eta_{1}, v_{1}\right)\right\|_{2}^{p} \leq K_{1}\left(1+\left\|\eta_{1}\right\|_{B_{h}^{\alpha}}^{p}+\left\|v_{1}\right\|^{p}\right) .
\end{aligned}
$$

$\left(\mathrm{A}_{2}^{*}\right)$ For any $\eta_{1}, \eta_{2} \in B_{h}^{\alpha}, v_{1}, v_{2} \in U$ and $0 \leq t \leq T$, there exists a constant $K_{2}$ such that

$$
\begin{aligned}
& \left|f\left(t, \eta_{1}, v_{1}\right)-f\left(t, \eta_{2}, v_{2}\right)\right|^{p}+\left\|g\left(t, \eta_{1}, v_{1}\right)-g\left(t, \eta_{2}, v_{2}\right)\right\|_{2}^{p} \\
& \quad \leq K_{2}\left(\left\|\eta_{1}-\eta_{2}\right\|_{B_{h}^{\alpha}}^{p}+\left\|v_{1}-v_{2}\right\|^{p}\right), \\
& \left|f\left(t, \eta_{1}, v_{1}\right)\right|^{p}+\left\|g\left(t, \eta_{1}, v_{1}\right)\right\|_{2}^{p} \leq K_{2} .
\end{aligned}
$$

$\left(\mathrm{A}_{3}\right)$ For each $0 \leq s<T$, the operator $\lambda\left(\lambda I+\Gamma_{s}^{T}\right)^{-1} \rightarrow 0$ in the strong operator topology as $\lambda \rightarrow 0^{+}$, where $\Gamma_{s}^{T}=\int_{s}^{T} S(T-r) B B^{*} S^{*}(T-r) d r$ is the controllability Grammian.

\section{Main results}

\subsection{Banach spaces $B_{h}$ and $B_{h}^{\alpha}$}

In this section we prove that $B_{h}$ and $B_{h}^{\alpha}$ are two Banach spaces and establish an important lemma, which will be used in the next section.

It is obvious that $\|\cdot\|_{B_{h}}$ is a norm. Following a similar discussion in [20] and [23], the following lemma can be obtained.

Lemma 3.1 For any $\varepsilon>0$ and $k>0$, there exists $\delta=\delta(\varepsilon, k)>0$ such that for any $\eta_{1}, \eta_{2} \in B_{h}$, if $\left\|\eta_{1}-\eta_{2}\right\|_{B_{h}} \leq \delta$, then $\sup _{-k \leq \tau \leq 0}\left|\eta_{1}(\tau)-\eta_{2}(\tau)\right| \leq \varepsilon$.

Lemma 3.2 Let $X_{a}=\left\{x \in X_{a}\right.$ is an $H$ valued continuous function defined on [-a,0], where $a$ is a positive constant and $\left.\sup _{-a \leq t \leq 0}|x(t)|<\infty\right\}$, then $X_{a}$ is a Banach space endowed with the norm $\|x\|=\sup _{-a \leq t \leq 0}|x(t)|$.

Lemma 3.3 $B_{h}$ is a Banach space.

Let $B_{T}=\left\{x \in B_{T} \mid x\right.$ is an $H$ valued continuous $\Im_{t}$ adapted process defined on $(-\infty, T]$, $E \sup _{0 \leq t \leq T}|x(t)|^{p}<\infty$ and $\left.x_{0}=A^{\alpha} \xi \in L^{p}\left(\Omega, B_{h}\right)\right\}$. Here $\Im_{t}:=\Im_{0}$ for all $t \leq 0$. We take a seminorm $\|\cdot\|_{T}$ defined by

$$
\|x\|_{T}=\left(E\left\|x_{0}\right\|_{B_{h}}^{p}\right)^{\frac{1}{p}}+\left(E \sup _{0 \leq t \leq T}|x(t)|^{p}\right)^{\frac{1}{p}} .
$$

In the following, an important lemma is established, which will play a crucial role in the development of the main results in the next section.

Lemma 3.4 Assume $x \in B_{T}$, then for $t \in[0, T], x_{t} \in L^{p}\left(\Omega, B_{h}\right)$, and

$$
l^{p} E|x(t)|^{p} \leq E\left\|x_{t}\right\|_{B_{h}}^{p} \leq 2^{p-1} l^{p} E \sup _{0 \leq s \leq t}|x(s)|^{p}+2^{p-1} E\left\|x_{0}\right\|_{B_{h}}^{p} .
$$


Proof For any $t \in[0, T]$, we have

$$
\begin{aligned}
& E\left\|x_{t}\right\|_{B_{h}}^{p}=E\left(\int_{-\infty}^{0} h(s) \sup _{s \leq \tau \leq 0}\left|x_{t}(\tau)\right| d s\right)^{p} \\
& =E\left(\int_{-\infty}^{-t} h(s) \sup _{s \leq \tau \leq 0}\left|x_{t}(\tau)\right| d s+\int_{-t}^{0} h(s) \sup _{s \leq \tau \leq 0}\left|x_{t}(\tau)\right| d s\right)^{p} \\
& =E\left(\int_{-\infty}^{-t} h(s) \sup _{t+s \leq \tau \leq t}|x(\tau)| d s+\int_{-t}^{0} h(s) \sup _{t+s \leq \tau \leq t}|x(\tau)| d s\right)^{p} \\
& \leq E\left(\int_{-\infty}^{-t} h(s)\left(\sup _{t+s \leq \tau \leq 0}|x(\tau)|+\sup _{0 \leq \tau \leq t}|x(\tau)|\right) d s+\int_{-t}^{0} h(s) \sup _{0 \leq \tau \leq t}|x(\tau)| d s\right)^{p} \\
& =E\left(\int_{-\infty}^{-t} h(s) \sup _{t+s \leq \tau \leq 0}|x(\tau)| d s+\int_{-\infty}^{0} h(s) d s \times \sup _{0 \leq \tau \leq t}|x(\tau)|\right)^{p} \\
& \leq E\left(\int_{-\infty}^{0} h(s) \sup _{s \leq \tau \leq 0}|x(\tau)| d s+l \sup _{0 \leq \tau \leq t}|x(\tau)|\right)^{p} \\
& =E\left(\int_{-\infty}^{0} h(s) \sup _{s \leq \tau \leq 0}\left|x_{0}(\tau)\right| d s+l \sup _{0 \leq \tau \leq t}|x(\tau)|\right)^{p} \\
& =E\left(l \sup _{0 \leq \tau \leq t}|x(\tau)|+\left\|x_{0}\right\|_{B_{h}}\right)^{p} \\
& \leq E\left(2^{p-1} l^{p}\left(\sup _{0 \leq \tau \leq t}|x(\tau)|\right)^{p}+2^{p-1}\left\|x_{0}\right\|_{B_{h}}^{p}\right) \\
& =2^{p-1} l^{p} E \sup _{0 \leq s \leq t}|x(s)|^{p}+2^{p-1} E\left\|x_{0}\right\|_{B_{h}}^{p},
\end{aligned}
$$

and $\left(E\left\|x_{t}\right\|_{B_{h}}^{p}\right)^{\frac{1}{p}} \leq 2^{\frac{p-1}{p}} l\left(E \sup _{0 \leq s \leq t}|x(s)|^{p}\right)^{\frac{1}{p}}+2^{\frac{p-1}{p}}\left(E\left\|x_{0}\right\|_{B_{h}}^{p}\right)^{\frac{1}{p}}$, then $x_{t} \in L^{p}\left(\Omega, B_{h}\right)$. Moreover,

$$
E\left\|x_{t}\right\|_{B_{h}}^{p}=E\left(\int_{-\infty}^{0} h(s) \sup _{s \leq \tau \leq 0}\left|x_{t}(\tau)\right| d s\right)^{p} \geq E\left|x_{t}(0)\right|^{p}\left(\int_{-\infty}^{0} h(s) d s\right)^{p}=l^{p} E|x(t)|^{p},
$$

and $\left(E\left\|x_{t}\right\|_{B_{h}}^{p}\right)^{\frac{1}{p}} \geq l\left(E|x(t)|^{p}\right)^{\frac{1}{p}}$. We complete the proof.

\subsection{Controllability results}

In the following, we give the controllability results.

Let $J=[0, T]$, define an operator $\Psi_{\lambda}$ on $B_{T} \times C\left(J, L^{p}(\Omega, \Im, P ; U)\right)$ by $\Psi_{\lambda}(Z, u)(t)=$ $\left(Z^{\lambda}(t), u^{\lambda}(t)\right)$ for $(Z, u) \in B_{T} \times C\left(J, L^{p}(\Omega, \Im, P ; U)\right)$. We prove that the terminal value of the system can approximate to $h^{*}$. Here, $h^{*} \in L^{p}(\Omega, \Im, P ; H)$ is arbitrary, and $h^{*}=E h^{*}+$ $\int_{0}^{T} \varphi(s) d w(s)$.

$$
\left\{\begin{aligned}
Z^{\lambda}(t)= & S(t) A^{\alpha} \xi(0)+\int_{0}^{t} A^{\alpha} S(t-s) B u^{\lambda}(s) d s+\int_{0}^{t} A^{\alpha} S(t-s) f\left(s, A^{-\alpha} Z_{s}, u(s)\right) d s \\
& +\int_{0}^{t} A^{\alpha} S(t-s) g\left(s, A^{-\alpha} Z_{s}, u(s)\right) d w(s), \quad t \in J, \\
Z^{\lambda}(t)= & A^{\alpha} \xi(t), \quad t \in(-\infty, 0], \\
u^{\lambda}(t)= & B^{*} S^{*}(T-t)\left(\lambda I+\Gamma_{0}^{T}\right)^{-1}(E h-S(T) \xi(0)) \\
& -B^{*} S^{*}(T-t) \int_{0}^{t}\left(\lambda I+\Gamma_{s}^{T}\right)^{-1} S(T-s) f\left(s, A^{-\alpha} Z_{s}, u(s)\right) d s \\
& -B^{*} S^{*}(T-t) \int_{0}^{t}\left(\lambda I+\Gamma_{s}^{T}\right)^{-1} \\
& \times\left[S(T-s) g\left(s, A^{-\alpha} Z_{s}, u(s)\right)-\varphi(s)\right] d w(s), \quad t \in J .
\end{aligned}\right.
$$


Let $B_{T}^{0}=\left\{x \mid x \in B_{T}, x_{0}=0 \in L^{p}\left(\Omega, B_{h}\right)\right\}$, and for any $x \in B_{T}^{0}$,

$$
\|x\|_{T}=\left(E\left\|x_{0}\right\|_{B_{h}}^{p}\right)^{\frac{1}{p}}+\left(E \sup _{0 \leq t \leq T}|x(t)|^{p}\right)^{\frac{1}{p}}=\left(E \sup _{0 \leq t \leq T}|x(t)|^{p}\right)^{\frac{1}{p}} .
$$

It is obvious that $B_{T}^{0}$ is a Banach space with the norm $\|\cdot\|_{T}=\left(E \sup _{0 \leq t \leq T}|x(t)|^{p}\right)^{\frac{1}{p}}$. For $\xi(t)$, we define

$$
\bar{\xi}(t)= \begin{cases}A^{\alpha} \xi(t), & t \in(-\infty, 0], \\ S(t) A^{\alpha} \xi(0), & t \in J,\end{cases}
$$

and $\bar{\xi}(t) \in L^{p}\left(\Omega, B_{h}\right)$. Let

$$
Y^{\lambda}(t)=Z^{\lambda}(t)-\bar{\xi}(t) \quad \text { and } \quad Y(t)=Z(t)-\bar{\xi}(t)
$$

then it can be easily concluded that $Y(t) \in B_{T}^{0}$. Define an operator $\Phi_{\lambda}$ on $B_{T}^{0} \times C\left(J, L^{P}(\Omega\right.$, $\Im, P ; U))$ by $\Phi_{\lambda}(Y, u)(t)=\left(Y^{\lambda}(t), u^{\lambda}(t)\right)$ for $(Y, u) \in B_{T}^{0} \times C\left(J, L^{P}(\Omega, \Im, P ; U)\right)$, where $B_{T}^{0} \times$ $C\left(J, L^{P}(\Omega, \Im, P ; U)\right)$ is a Banach space with the norm $\|\cdot\|=\|Y\|_{T}+\|u\|$. Then from the above definition of $\left(Z^{\lambda}, u^{\lambda}\right)$, it holds that

$$
\left\{\begin{aligned}
Y^{\lambda}(t)= & \int_{0}^{t} A^{\alpha} S(t-s) B u^{\lambda}(s) d s+\int_{0}^{t} A^{\alpha} S(t-s) f\left(s, A^{-\alpha}\left(Y_{s}+\bar{\xi}_{s}\right), u(s)\right) d s \\
& +\int_{0}^{t} A^{\alpha} S(t-s) g\left(s, A^{-\alpha}\left(Y_{s}+\bar{\xi}_{s}\right), u(s)\right) d w(s), \quad t \in J, \\
Y^{\lambda}(t)= & 0, \quad t \in(-\infty, 0], \\
u^{\lambda}(t)= & B^{*} S^{*}(T-t)\left(\lambda I+\Gamma_{0}^{T}\right)^{-1}(E h-S(T) \xi(0)) \\
& -B^{*} S^{*}(T-t) \int_{0}^{t}\left(\lambda I+\Gamma_{s}^{T}\right)^{-1} S(T-s) f\left(s, A^{-\alpha}\left(Y_{s}+\bar{\xi}_{s}\right), u(s)\right) d s \\
& -B^{*} S^{*}(T-t) \int_{0}^{t}\left(\lambda I+\Gamma_{s}^{T}\right)^{-1} \\
& \times\left[S(T-s) g\left(s, A^{-\alpha}\left(Y_{s}+\bar{\xi}_{s}\right), u(s)\right)-\varphi(s)\right] d w(s), \quad t \in J .
\end{aligned}\right.
$$

By Lemma 3.4, we also remark that for any $t \in J$,

$$
\begin{aligned}
E\left\|A^{-\alpha}\left(Y_{t}+\bar{\xi}_{t}\right)\right\|_{B_{h}^{\alpha}}^{p}= & E\left\|Y_{t}+\bar{\xi}_{t}\right\|_{B_{h}}^{p} \\
\leq & E\left(\left\|Y_{t}\right\|_{B_{h}}+\left\|\bar{\xi}_{t}\right\|_{B_{h}}\right)^{p} \\
\leq & 2^{p-1} E\left\|Y_{t}\right\|_{B_{h}}^{p}+2^{p-1} E\left\|\bar{\xi}_{t}\right\|_{B_{h}}^{p} \\
\leq & 4^{p-1} E\left\|Y_{0}\right\|_{B_{h}}^{p}+4^{p-1} E\left\|\bar{\xi}_{0}\right\|_{B_{h}}^{p}+4^{p-1} l^{p} E \sup _{0 \leq s \leq t}|Y(s)|^{p} \\
& +4^{p-1} l^{p} E \sup _{0 \leq s \leq t}|\bar{\xi}(s)|^{p} \\
& \leq 4^{p-1}\left(E\|\xi\|_{B_{h}^{\alpha}}^{p}+l^{p} E \sup _{0 \leq t \leq T}|Y(t)|^{p}+l^{p} E \sup _{0 \leq t \leq T}\left|S(t) A^{\alpha} \xi(0)\right|^{p}\right) \\
\leq & 4^{p-1}\left(E\|\xi\|_{B_{h}^{\alpha}}^{p}+l^{p}\|Y\|_{T}^{p}+l^{p} M^{p} E\left|A^{\alpha} \xi(0)\right|^{p}\right) .
\end{aligned}
$$

It can be seen that the operator $\Psi_{\lambda}$ has a fixed point on $B_{T} \times C\left(J, L^{p}(\Omega, \Im, P ; U)\right)$ if and only if the operator $\Phi_{\lambda}$ has a fixed point on $B_{T}^{0} \times C\left(J, L^{p}(\Omega, \Im, P ; U)\right)$. 
Lemma 3.5 Let $0<\alpha<\frac{p-2}{2 p}$, and $\left(\mathrm{A}_{1}\right),\left(\mathrm{A}_{2}\right)$ hold, then for any $\lambda>0$, the operator $\Phi_{\lambda}$ maps $B_{T}^{0} \times C\left(J, L^{P}(\Omega, \Im, P ; U)\right)$ into $B_{T}^{0} \times C\left(J, L^{P}(\Omega, \Im, P ; U)\right)$.

Proof We just need to prove that $Y^{\lambda}(t)$ and $u^{\lambda}(t)$ are bounded on $J$ and continuous in $L^{p}$ sense on $J$. By $\left(\mathrm{A}_{1}\right),\left(\mathrm{A}_{2}\right)$, the Hölder inequality and the Burkholder-Davis-Gundy inequality, we have

$$
\begin{aligned}
E\left\|u^{\lambda}(t)\right\|^{p}= & E \| B^{*} S^{*}(T-t)\left(\lambda I+\Gamma_{0}^{T}\right)^{-1}(E h-S(T) \xi(0)) \\
& -B^{*} S^{*}(T-t) \int_{0}^{t}\left(\lambda I+\Gamma_{s}^{T}\right)^{-1} S(T-s) f\left(s, A^{-\alpha}\left(Y_{s}+\bar{\xi}_{s}\right), u(s)\right) d s \\
& -B^{*} S^{*}(T-t) \int_{0}^{t}\left(\lambda I+\Gamma_{s}^{T}\right)^{-1} \\
& \times\left[S(T-s) g\left(s, A^{-\alpha}\left(Y_{s}+\bar{\xi}_{s}\right), u(s)\right)-\varphi(s)\right] d w(s) \|^{p} \\
\leq & 5^{p-1}\left\|B^{*} S^{*}(T-t)\left(\lambda I+\Gamma_{0}^{T}\right)^{-1} E h\right\|^{p} \\
& +5^{p-1} E\left\|B^{*} S^{*}(T-t)\left(\lambda I+\Gamma_{0}^{T}\right)^{-1} S(T) \xi(0)\right\|^{p} \\
& +5^{p-1} E\left\|B^{*} S^{*}(T-t) \int_{0}^{t}\left(\lambda I+\Gamma_{s}^{T}\right)^{-1} S(T-s) f\left(s, A^{-\alpha}\left(Y_{s}+\bar{\xi}_{s}\right), u(s)\right) d s\right\|^{p} \\
& +5^{p-1} E \| B^{*} S^{*}(T-t) \int_{0}^{t}\left(\lambda I+\Gamma_{s}^{T}\right)^{-1} S(T-s) \\
& \times g\left(s, A^{-\alpha}\left(Y_{s}+\bar{\xi}_{s}\right), u(s)\right) d w(s) \|^{p} \\
& +5^{p-1} E\left\|B^{*} S^{*}(T-t) \int_{0}^{t}\left(\lambda I+\Gamma_{s}^{T}\right)^{-1} S(T-s) \varphi(s) d w(s)\right\|^{p} \\
= & I_{1}+I_{2}+I_{3}+I_{4}+I_{5} .
\end{aligned}
$$

For each one

$$
\begin{aligned}
I_{1} & =5^{p-1}\left\|B^{*} S^{*}(T-t)\left(\lambda I+\Gamma_{0}^{T}\right)^{-1} E h\right\|^{p} \leq 5^{p-1}\|B\|^{p} M^{p} \frac{1}{\lambda^{p}}|E h|^{p}, \\
I_{2} & =5^{p-1} E\left\|B^{*} S^{*}(T-t)\left(\lambda I+\Gamma_{0}^{T}\right)^{-1} S(T) \xi(0)\right\|^{p} \leq 5^{p-1}\|B\|^{p} M^{2 p} \frac{1}{\lambda^{p}} E|\xi(0)|^{p}, \\
I_{3} & =5^{p-1} E\left\|B^{*} S^{*}(T-t) \int_{0}^{t}\left(\lambda I+\Gamma_{s}^{T}\right)^{-1} S(T-s) f\left(s, A^{-\alpha}\left(Y_{s}+\bar{\xi}_{s}\right), u(s)\right) d s\right\|^{p} \\
& \leq 5^{p-1}\|B\|^{p} M^{2 p} \frac{1}{\lambda^{p}} T^{p-1} E \int_{0}^{t}\left|f\left(s, A^{-\alpha}\left(Y_{s}+\bar{\xi}_{s}\right), u(s)\right)\right|^{p} d s \\
& \leq 5^{p-1}\|B\|^{p} M^{2 p} \frac{1}{\lambda^{p}} T^{p-1} \int_{0}^{t} K_{1}\left(1+E\left\|A^{-\alpha}\left(Y_{s}+\bar{\xi}_{s}\right)\right\|_{B_{h}^{\alpha}}^{p}+E\|u(s)\|^{p}\right) d s \\
& \leq 5^{p-1}\|B\|^{p} M^{2 p} \frac{1}{\lambda^{p}} T^{p} K_{1}\left(1+4^{p-1}\left(E\|\xi\|_{B_{h}^{\alpha}}^{p}+l^{p}\|Y\|_{T}^{p}+l^{p} M^{p} E\left|A^{\alpha} \xi(0)\right|^{p}\right)+\|u\|^{p}\right), \\
I_{4} & =5^{p-1} E\left\|B^{*} S^{*}(T-t) \int_{0}^{t}\left(\lambda I+\Gamma_{s}^{T}\right)^{-1} S(T-s) g\left(s, A^{-\alpha}\left(Y_{s}+\bar{\xi}_{s}\right), u(s)\right) d w(s)\right\|^{p} \\
& \leq 5^{p-1}\|B\|^{p} M^{p} C_{p} E\left(\int_{0}^{t}\left\|\left(\lambda I+\Gamma_{s}^{T}\right)^{-1} S(T-s)\right\|^{2}\left\|g\left(s, A^{-\alpha}\left(Y_{s}+\bar{\xi}_{s}\right), u(s)\right)\right\|_{2}^{2} d s\right)^{\frac{p}{2}}
\end{aligned}
$$




$$
\begin{aligned}
& \leq 5^{p-1}\|B\|^{p} M^{2 p} C_{p} \frac{1}{\lambda^{p}} T^{\frac{p}{2}-1} E \int_{0}^{t}\left\|g\left(s, A^{-\alpha}\left(Y_{s}+\bar{\xi}_{s}\right), u(s)\right)\right\|_{2}^{p} d s \\
& \leq 5^{p-1}\|B\|^{p} M^{2 p} C_{p} \frac{1}{\lambda^{p}} T^{\frac{p}{2}-1} \int_{0}^{t} K_{1}\left(1+E\left\|A^{-\alpha}\left(Y_{s}+\bar{\xi}_{s}\right)\right\|_{B_{h}^{\alpha}}^{p}+E\|u(s)\|^{p}\right) d s \\
& \leq 5^{p-1}\|B\|^{p} M^{2 p} C_{p} \frac{1}{\lambda^{p}} T^{\frac{p}{2}} K_{1}\left(1+4^{p-1}\left(E\|\xi\|_{B_{h}^{\alpha}}^{p}+l^{p}\|Y\|_{T}^{p}+l^{p} M^{p} E\left|A^{\alpha} \xi(0)\right|^{p}\right)+\|u\|^{p}\right) \\
I_{5} & =5^{p-1} E\left\|B^{*} S^{*}(T-t) \int_{0}^{t}\left(\lambda I+\Gamma_{s}^{T}\right)^{-1} S(T-s) \varphi(s) d w(s)\right\|^{p} \\
& \leq 5^{p-1}\|B\|^{p} M^{p} C_{p} E\left(\int_{0}^{t}\left\|\left(\lambda I+\Gamma_{s}^{T}\right)^{-1} S(T-s)\right\|^{2}\|\varphi(s)\|_{2}^{2} d s\right)^{\frac{p}{2}} \\
& \leq 5^{p-1}\|B\|^{p} M^{2 p} C_{p} \frac{1}{\lambda^{p}} T^{\frac{p}{2}-1} E\left(\int_{0}^{T}\|\varphi(s)\|_{2}^{2} d s\right)^{\frac{p}{2}} .
\end{aligned}
$$

For $t \in J$ is arbitrary, we can easily obtain that $\sup _{0 \leq t \leq T} E\left\|u^{\lambda}(t)\right\|^{p}<\infty$. On the other hand, set $t_{2}>t_{1} \geq 0$ for $u^{\lambda}\left(t_{2}\right)$ and $u^{\lambda}\left(t_{1}\right)$, we have

$$
\begin{aligned}
& E\left\|u^{\lambda}\left(t_{2}\right)-u^{\lambda}\left(t_{1}\right)\right\|^{p} \\
& \leq 5^{p-1}\left\|B^{*}\left(S^{*}\left(T-t_{2}\right)-S^{*}\left(T-t_{1}\right)\right)\left(\lambda I+\Gamma_{0}^{T}\right)^{-1} E h\right\|^{p} \\
&+5^{p-1} E\left\|B^{*}\left(S^{*}\left(T-t_{2}\right)-S^{*}\left(T-t_{1}\right)\right)\left(\lambda I+\Gamma_{0}^{T}\right)^{-1} S(T) \xi(0)\right\|^{p} \\
&+5^{p-1} E \| B^{*} S^{*}\left(T-t_{2}\right) \int_{0}^{t_{2}}\left(\lambda I+\Gamma_{s}^{T}\right)^{-1} S(T-s) f\left(s, A^{-\alpha}\left(Y_{s}+\bar{\xi}_{s}\right), u(s)\right) d s \\
&-B^{*} S^{*}\left(T-t_{1}\right) \int_{0}^{t_{1}}\left(\lambda I+\Gamma_{s}^{T}\right)^{-1} S(T-s) f\left(s, A^{-\alpha}\left(Y_{s}+\bar{\xi}_{s}\right), u(s)\right) d s \|^{p} \\
&+5^{p-1} E \| B^{*} S^{*}\left(T-t_{2}\right) \int_{0}^{t_{2}}\left(\lambda I+\Gamma_{s}^{T}\right)^{-1} S(T-s) g\left(s, A^{-\alpha}\left(Y_{s}+\bar{\xi}_{s}\right), u(s)\right) d w(s) \\
&-B^{*} S^{*}\left(T-t_{1}\right) \int_{0}^{t_{1}}\left(\lambda I+\Gamma_{s}^{T}\right)^{-1} S(T-s) g\left(s, A^{-\alpha}\left(Y_{s}+\bar{\xi}_{s}\right), u(s)\right) d w(s) \|^{p} \\
&+5^{p-1} E \| B^{*} S^{*}\left(T-t_{2}\right) \int_{0}^{t_{2}}\left(\lambda I+\Gamma_{s}^{T}\right)^{-1} S(T-s) \varphi(s) d w(s) \\
&-B^{*} S^{*}\left(T-t_{1}\right) \int_{0}^{t_{1}}\left(\lambda I+\Gamma_{s}^{T}\right)^{-1} S(T-s) \varphi(s) d w(s) \|^{p} \\
&= I_{11}+I_{12}+I_{13}+I_{14}+I_{15},
\end{aligned}
$$

and

$$
\begin{aligned}
I_{11} & =5^{p-1}\left\|B^{*}\left(S^{*}\left(T-t_{2}\right)-S^{*}\left(T-t_{1}\right)\right)\left(\lambda I+\Gamma_{0}^{T}\right)^{-1} E h\right\|^{p} \\
& =5^{p-1}\left\|B^{*} S^{*}\left(T-t_{2}\right)\left(S^{*}\left(t_{2}-t_{1}\right)-I\right)\left(\lambda I+\Gamma_{0}^{T}\right)^{-1} E h\right\|^{p} \\
& \leq 5^{p-1}\|B\|^{p} M^{p} \frac{1}{\lambda^{p}}|E h|^{p}\left\|S^{*}\left(t_{2}-t_{1}\right)-I\right\|^{p}, \\
I_{12} & =5^{p-1} E\left\|B^{*}\left(S^{*}\left(T-t_{2}\right)-S^{*}\left(T-t_{1}\right)\right)\left(\lambda I+\Gamma_{0}^{T}\right)^{-1} S(T) \xi(0)\right\|^{p} \\
& =5^{p-1} E\left\|B^{*} S^{*}\left(T-t_{2}\right)\left(S^{*}\left(t_{2}-t_{1}\right)-I\right)\left(\lambda I+\Gamma_{0}^{T}\right)^{-1} S(T) \xi(0)\right\|^{p} \\
& \leq 5^{p-1}\|B\|^{p} M^{2 p} \frac{1}{\lambda^{p}} E|\xi(0)|^{p}\left\|S^{*}\left(t_{2}-t_{1}\right)-I\right\|^{p},
\end{aligned}
$$




$$
\begin{aligned}
& I_{13}=5^{p-1} E \| B^{*} S^{*}\left(T-t_{2}\right) \int_{0}^{t_{2}}\left(\lambda I+\Gamma_{s}^{T}\right)^{-1} S(T-s) f\left(s, A^{-\alpha}\left(Y_{s}+\bar{\xi}_{s}\right), u(s)\right) d s \\
& -B^{*} S^{*}\left(T-t_{1}\right) \int_{0}^{t_{1}}\left(\lambda I+\Gamma_{s}^{T}\right)^{-1} S(T-s) f\left(s, A^{-\alpha}\left(Y_{s}+\bar{\xi}_{s}\right), u(s)\right) d s \|^{p} \\
& =5^{p-1} E \| B^{*} S^{*}\left(T-t_{2}\right) \int_{t_{1}}^{t_{2}}\left(\lambda I+\Gamma_{s}^{T}\right)^{-1} S(T-s) f\left(s, A^{-\alpha}\left(Y_{s}+\bar{\xi}_{s}\right), u(s)\right) d s \\
& +B^{*}\left(S^{*}\left(T-t_{2}\right)-S^{*}\left(T-t_{1}\right)\right) \int_{0}^{t_{1}}\left(\lambda I+\Gamma_{s}^{T}\right)^{-1} S(T-s) f\left(s, A^{-\alpha}\left(Y_{s}+\bar{\xi}_{s}\right), u(s)\right) d s \|^{p} \\
& \leq 10^{p-1} E\left\|B^{*} S^{*}\left(T-t_{2}\right) \int_{t_{1}}^{t_{2}}\left(\lambda I+\Gamma_{s}^{T}\right)^{-1} S(T-s) f\left(s, A^{-\alpha}\left(Y_{s}+\bar{\xi}_{s}\right), u(s)\right) d s\right\|^{p} \\
& +10^{p-1} E \| B^{*}\left(S^{*}\left(T-t_{2}\right)-S^{*}\left(T-t_{1}\right)\right) \\
& \times \int_{0}^{t_{1}}\left(\lambda I+\Gamma_{s}^{T}\right)^{-1} S(T-s) f\left(s, A^{-\alpha}\left(Y_{s}+\bar{\xi}_{s}\right), u(s)\right) d s \|^{p} \\
& \leq 10^{p-1}\|B\|^{p} M^{2 p} \frac{1}{\lambda^{p}}\left(t_{2}-t_{1}\right)^{p} K_{1}\left(1+4^{p-1}\left(E\|\xi\|_{B_{h}^{\alpha}}^{p}+l^{p}\|Y\|_{T}^{p}\right.\right. \\
& \left.\left.+l^{p} M^{p} E\left|A^{\alpha} \xi(0)\right|^{p}\right)+\|u\|^{p}\right)+10^{p-1}\|B\|^{p} M^{2 p} \frac{1}{\lambda^{p}} T^{p} \\
& \times K_{1}\left(1+4^{p-1}\left(E\|\xi\|_{B_{h}^{\alpha}}^{p}+l^{p}\|Y\|_{T}^{p}+l^{p} M^{p} E\left|A^{\alpha} \xi(0)\right|^{p}\right)+\|u\|^{p}\right)\left\|S^{*}\left(t_{2}-t_{1}\right)-I\right\|^{p}, \\
& I_{14}=5^{p-1} E \| B^{*} S^{*}\left(T-t_{2}\right) \int_{0}^{t_{2}}\left(\lambda I+\Gamma_{s}^{T}\right)^{-1} S(T-s) g\left(s, A^{-\alpha}\left(Y_{s}+\bar{\xi}_{s}\right), u(s)\right) d w(s) \\
& -B^{*} S^{*}\left(T-t_{1}\right) \int_{0}^{t_{1}}\left(\lambda I+\Gamma_{s}^{T}\right)^{-1} S(T-s) g\left(s, A^{-\alpha}\left(Y_{s}+\bar{\xi}_{s}\right), u(s)\right) d w(s) \|^{p} \\
& \leq 10^{p-1} E\left\|B^{*} S^{*}\left(T-t_{2}\right) \int_{t_{1}}^{t_{2}}\left(\lambda I+\Gamma_{s}^{T}\right)^{-1} S(T-s) g\left(s, A^{-\alpha}\left(Y_{s}+\bar{\xi}_{s}\right), u(s)\right) d w(s)\right\|^{p} \\
& +10^{p-1} E \| B^{*}\left(S^{*}\left(T-t_{2}\right)-S^{*}\left(T-t_{1}\right)\right) \\
& \times \int_{0}^{t_{1}}\left(\lambda I+\Gamma_{s}^{T}\right)^{-1} S(T-s) g\left(s, A^{-\alpha}\left(Y_{s}+\bar{\xi}_{s}\right), u(s)\right) d w(s) \|^{p} \\
& \leq 10^{p-1} C_{p}\|B\|^{p} M^{2 p} \frac{1}{\lambda^{p}}\left(t_{2}-t_{1}\right)^{\frac{p}{2}} K_{1}\left(1+4^{p-1}\left(E\|\xi\|_{B_{h}^{\alpha}}^{p}+l^{p}\|Y\|_{T}^{p}\right.\right. \\
& \left.\left.+l^{p} M^{p} E\left|A^{\alpha} \xi(0)\right|^{p}\right)+\|u\|^{p}\right)+10^{p-1} C_{p}\|B\|^{p} M^{2 p} \frac{1}{\lambda^{p}} T^{\frac{p}{2}} \\
& \times K_{1}\left(1+4^{p-1}\left(E\|\xi\|_{B_{h}^{\alpha}}^{p}+l^{p}\|Y\|_{T}^{p}+l^{p} M^{p} E\left|A^{\alpha} \xi(0)\right|^{p}\right)+\|u\|^{p}\right)\left\|S^{*}\left(t_{2}-t_{1}\right)-I\right\|^{p}, \\
& I_{15}=5^{p-1} E \| B^{*} S^{*}\left(T-t_{2}\right) \int_{0}^{t_{2}}\left(\lambda I+\Gamma_{s}^{T}\right)^{-1} S(T-s) \varphi(s) d w(s) \\
& -B^{*} S^{*}\left(T-t_{1}\right) \int_{0}^{t_{1}}\left(\lambda I+\Gamma_{s}^{T}\right)^{-1} S(T-s) \varphi(s) d w(s) \|^{p} \\
& \leq 10^{p-1} E\left\|B^{*} S^{*}\left(T-t_{2}\right) \int_{t_{1}}^{t_{2}}\left(\lambda I+\Gamma_{s}^{T}\right)^{-1} S(T-s) \varphi(s) d w(s)\right\|^{p} \\
& +10^{p-1} E\left\|B^{*}\left(S^{*}\left(T-t_{2}\right)-S^{*}\left(T-t_{1}\right)\right) \int_{0}^{t_{1}}\left(\lambda I+\Gamma_{s}^{T}\right)^{-1} S(T-s) \varphi(s) d w(s)\right\|^{p}
\end{aligned}
$$




$$
\begin{aligned}
\leq & 10^{p-1} C_{p}\|B\|^{p} M^{2 p} \frac{1}{\lambda^{p}}\left(t_{2}-t_{1}\right)^{\frac{p}{2}-1} E\left(\int_{0}^{T}\|\varphi(s)\|_{2}^{2} d s\right)^{\frac{p}{2}} \\
& +10^{p-1} C_{p}\|B\|^{p} M^{2 p} \frac{1}{\lambda^{p}} T^{\frac{p}{2}-1} E\left(\int_{0}^{T}\|\varphi(s)\|_{2}^{2} d s\right)^{\frac{p}{2}}\left\|S^{*}\left(t_{2}-t_{1}\right)-I\right\|^{p} .
\end{aligned}
$$

From the above equations, we can obtain that $E\left|u^{\lambda}\left(t_{2}\right)-u^{\lambda}\left(t_{1}\right)\right|^{p} \rightarrow 0$ as $t_{1} \rightarrow t_{2}$. Then $u^{\lambda}(t)$ is continuous in $L^{p}$ sense on $J$ and $u^{\lambda}(t) \in C\left(J, L^{p}(\Omega, \Im, P ; U)\right)$. Moreover, for $Y^{\lambda}(t)$ and $t \geq 0$, we have

$$
\begin{aligned}
E \sup _{0 \leq s \leq t}\left|Y^{\lambda}(s)\right|^{p}= & E \sup _{0 \leq s \leq t} \mid \int_{0}^{s} A^{\alpha} S(s-r) B u^{\lambda}(r) d r \\
& +\int_{0}^{s} A^{\alpha} S(s-r) f\left(r, A^{-\alpha}\left(Y_{r}+\bar{\xi}_{r}\right), u(r)\right) d r \\
& +\left.\int_{0}^{s} A^{\alpha} S(s-r) g\left(r, A^{-\alpha}\left(Y_{r}+\bar{\xi}_{r}\right), u(r)\right) d w(r)\right|^{p} \\
\leq & 3^{p-1} E \sup _{0 \leq s \leq t}\left|\int_{0}^{s} A^{\alpha} S(s-r) B u^{\lambda}(r) d r\right|^{p} \\
& +3^{p-1} E \sup _{0 \leq s \leq t}\left|\int_{0}^{s} A^{\alpha} S(s-r) f\left(r, A^{-\alpha}\left(Y_{r}+\bar{\xi}_{r}\right), u(r)\right) d r\right|^{p} \\
& +3^{p-1} E \sup _{0 \leq s \leq t}\left|\int_{0}^{s} A^{\alpha} S(s-r) g\left(r, A^{-\alpha}\left(Y_{r}+\bar{\xi}_{r}\right), u(r)\right) d w(r)\right|^{p} \\
\leq & I_{21}+I_{22}+I_{23} .
\end{aligned}
$$

Let $\frac{1}{p}+\frac{1}{q}=1$. From Lemma 2.7, it can be obtained that

$$
\begin{aligned}
I_{21} & =3^{p-1} E \sup _{0 \leq s \leq t}\left|\int_{0}^{s} A^{\alpha} S(s-r) B u^{\lambda}(r) d r\right|^{p} \\
& \leq 3^{p-1} E \sup _{0 \leq s \leq t}\left(\int_{0}^{s}\left|A^{\alpha} S(s-r) B u^{\lambda}(r)\right| d r\right)^{p} \\
& \leq 3^{p-1} E \sup _{0 \leq s \leq t}\left(\int_{0}^{s} M_{\alpha} e^{-a(s-r)}(s-r)^{-\alpha}\left|B u^{\lambda}(r)\right| d r\right)^{p} \\
& \leq 3^{p-1}\|B\|^{p} M_{\alpha}^{p} E \sup _{0 \leq s \leq t}\left(\int_{0}^{s}(s-r)^{-\alpha q} d s\right)^{\frac{p}{q}} \int_{0}^{s}\left\|u^{\lambda}(r)\right\|^{p} d r \\
& =3^{p-1}\|B\|^{p} M_{\alpha}^{p} E \sup _{0 \leq s \leq t}\left(\frac{s^{1-\alpha q}}{1-\alpha q}\right)^{\frac{p}{q}} \int_{0}^{s}\left\|u^{\lambda}(r)\right\|^{p} d r \\
& =3^{p-1}\|B\|^{p} M_{\alpha}^{p} E\left(\frac{t^{1-\alpha q}}{1-\alpha q}\right)^{\frac{p}{q}} \int_{0}^{t}\left\|u^{\lambda}(s)\right\|^{p} d s \\
& \leq 3^{p-1}\|B\|^{p} M_{\alpha}^{p}\left(\frac{T^{1-\alpha q}}{1-\alpha q}\right)^{\frac{p}{q}} T\left\|u^{\lambda}\right\|^{p}, \\
I_{22} & =3^{p-1} E \sup _{0 \leq s \leq t}\left|\int_{0}^{s} A^{\alpha} S(s-r) f\left(r, A^{-\alpha}\left(Y_{r}+\bar{\xi}_{r}\right), u(r)\right) d r\right|^{p} \\
& \leq 3^{p-1} E \sup _{0 \leq s \leq t}\left(\int_{0}^{s}\left|A^{\alpha} S(s-r) f\left(r, A^{-\alpha}\left(Y_{r}+\bar{\xi}_{r}\right), u(r)\right)\right| d r\right)^{p}
\end{aligned}
$$




$$
\begin{aligned}
& \leq 3^{p-1} E \sup _{0 \leq s \leq t}\left(\int_{0}^{s} M_{\alpha} e^{-a(s-r)}(s-r)^{-\alpha}\left|f\left(r, A^{-\alpha}\left(Y_{r}+\bar{\xi}_{r}\right), u(r)\right)\right| d r\right)^{p} \\
& \leq 3^{p-1} M_{\alpha}^{p}\left(\int_{0}^{t}(t-s)^{-\alpha q} d s\right)^{\frac{p}{q}} \int_{0}^{t} E\left|f\left(s, A^{-\alpha}\left(Y_{s}+\bar{\xi}_{s}\right), u(s)\right)\right|^{p} d s \\
& \leq 3^{p-1} M_{\alpha}^{p}\left(\frac{T^{1-\alpha q}}{1-\alpha q}\right)^{\frac{p}{q}} T K_{1}\left(1+4^{p-1}\left(E\|\xi\|_{B_{h}^{\alpha}}^{p}+l^{p}\|Y\|_{T}^{p}+l^{p} M^{p} E\left|A^{\alpha} \xi(0)\right|^{p}\right)+\|u\|^{p}\right), \\
I_{23}= & 3^{p-1} E \sup _{0 \leq s \leq t}\left|\int_{0}^{s} A^{\alpha} S(s-r) g\left(r, A^{-\alpha}\left(Y_{r}+\bar{\xi}_{r}\right), u(r)\right) d w(r)\right|^{p} \\
\leq & 3^{p-1} C_{p} E\left(\int_{0}^{t}\left\|A^{\alpha} S(t-s) g\left(s, A^{-\alpha}\left(Y_{s}+\bar{\xi}_{s}\right), u(s)\right)\right\|_{2}^{2} d s\right)^{\frac{p}{2}} \\
\leq & 3^{p-1} C_{p} E\left(\int_{0}^{t} M_{\alpha}^{2} e^{-2 a(t-s)}(t-s)^{-2 \alpha}\left\|g\left(s, A^{-\alpha}\left(Y_{s}+\bar{\xi}_{s}\right), u(s)\right)\right\|_{2}^{2} d s\right)^{\frac{p}{2}} \\
\leq & 3^{p-1} C_{p} M_{\alpha}^{p}\left(\frac{t^{1-\frac{2 \alpha p}{p-2}}}{1-\frac{2 \alpha p}{p-2}}\right)^{\frac{p-2}{2}} \int_{0}^{t} E\left\|g\left(s, A^{-\alpha}\left(Y_{s}+\bar{\xi}_{s}\right), u(s)\right)\right\|^{p} d s \\
\leq & 3^{p-1} C_{p} M_{\alpha}^{p}\left(\frac{T^{1-\frac{2 \alpha p}{p-2}}}{1-\frac{2 \alpha p}{p-2}}\right)^{\frac{p-2}{2}} \\
& \times T K_{1}\left(1+4^{p-1}\left(E\|\xi\|_{B_{h}^{\alpha}}^{p}+l^{p}\|Y\|_{T}^{p}+l^{p} M^{p} E\left|A^{\alpha} \xi(0)\right|^{p}\right)+\|u\|^{p}\right) .
\end{aligned}
$$

Then it can be obtained that $E \sup _{0 \leq t \leq T}\left|Y^{\lambda}(t)\right|^{p}<\infty$. Meanwhile, for $Y^{\lambda}\left(t_{1}\right)$ and $Y^{\lambda}\left(t_{2}\right)$, we have

$$
\begin{aligned}
E\left|Y^{\lambda}\left(t_{2}\right)-Y^{\lambda}\left(t_{1}\right)\right|^{p} & \\
\leq & 3^{p-1} E\left|\int_{0}^{t_{2}} A^{\alpha} S\left(t_{2}-s\right) B u^{\lambda}(s) d s-\int_{0}^{t_{1}} A^{\alpha} S\left(t_{1}-s\right) B u^{\lambda}(s) d s\right|^{p} \\
& +3^{p-1} E \mid \int_{0}^{t_{2}} A^{\alpha} S\left(t_{2}-s\right) f\left(s, A^{-\alpha}\left(Y_{s}+\bar{\xi}_{s}\right), u(s)\right) d s \\
& -\left.\int_{0}^{t_{1}} A^{\alpha} S\left(t_{1}-s\right) f\left(s, A^{-\alpha}\left(Y_{s}+\bar{\xi}_{s}\right), u(s)\right) d s\right|^{p} \\
& +3^{p-1} E \mid \int_{0}^{t_{2}} A^{\alpha} S\left(t_{2}-s\right) g\left(s, A^{-\alpha}\left(Y_{s}+\bar{\xi}_{s}\right), u(s)\right) d w(s) \\
& -\left.\int_{0}^{t_{1}} A^{\alpha} S\left(t_{1}-s\right) g\left(s, A^{-\alpha}\left(Y_{s}+\bar{\xi}_{s}\right), u(s)\right) d w(s)\right|^{p} \\
= & I_{31}+I_{32}+I_{33},
\end{aligned}
$$

and

$$
\begin{aligned}
I_{31} & =3^{p-1} E\left|\int_{0}^{t_{2}} A^{\alpha} S\left(t_{2}-s\right) B u^{\lambda}(s) d s-\int_{0}^{t_{1}} A^{\alpha} S\left(t_{1}-s\right) B u^{\lambda}(s) d s\right|^{p} \\
& \leq 6^{p-1} E\left|\int_{t_{1}}^{t_{2}} A^{\alpha} S\left(t_{2}-s\right) B u^{\lambda}(s) d s\right|^{p}+6^{p-1} E\left|\int_{0}^{t_{1}} A^{\alpha}\left(S\left(t_{2}-s\right)-S\left(t_{1}-s\right)\right) B u^{\lambda}(s) d s\right|^{p} \\
& \leq 6^{p-1} M_{\alpha}^{p}\|B\|^{p} E\left(\int_{t_{1}}^{t_{2}}\left(t_{2}-s\right)^{-\alpha} e^{-a\left(t_{2}-s\right)}\left\|u^{\lambda}(s)\right\| d s\right)^{p}
\end{aligned}
$$




$$
\begin{aligned}
& +6^{p-1} M_{\alpha}^{p} E\left(\int_{0}^{t_{1}}\left(\frac{t_{1}-s}{2}\right)^{-\alpha} e^{-a\left(\frac{t_{1}-s}{2}\right)}\left\|\left(S\left(t_{2}-t_{1}\right)-I\right) S\left(\frac{t_{1}-s}{2}\right) B u^{\lambda}(s)\right\| d s\right)^{p} \\
& \leq 6^{p-1} M_{\alpha}^{p}\|B\|^{p}\left(\int_{t_{1}}^{t_{2}}\left(t_{2}-s\right)^{-\alpha q} e^{-a q\left(t_{2}-s\right)} d s\right)^{\frac{p}{q}} E \int_{t_{1}}^{t_{2}}\left\|u^{\lambda}(s)\right\|^{p} d s \\
& +6^{p-1} M_{\alpha}^{p} E\left(\int_{0}^{t_{1}}\left(\frac{t_{1}-s}{2}\right)^{-\alpha} e^{-a\left(\frac{t_{1}-s}{2}\right)}\left\|\left(S\left(t_{2}-t_{1}\right)-I\right) S\left(\frac{t_{1}-s}{2}\right) B u^{\lambda}(s)\right\| d s\right)^{p} \\
& \leq 6^{p-1} M_{\alpha}^{p}\|B\|^{p}\left(\int_{0}^{t_{2}}\left(t_{2}-s\right)^{-\alpha q} d s\right)^{\frac{p}{q}}\left(t_{2}-t_{1}\right)\left\|u^{\lambda}\right\|^{p} \\
& +6^{p-1} M_{\alpha}^{p} E\left(\int_{0}^{t_{1}}\left(\frac{t_{1}-s}{2}\right)^{-\alpha} e^{-a\left(\frac{t_{1}-s}{2}\right)} N_{\alpha}\left(t_{2}-t_{1}\right)^{\alpha}\left|A^{\alpha} S\left(\frac{t_{1}-s}{2}\right) B u^{\lambda}(s)\right| d s\right)^{p} \\
& \leq 6^{p-1} M_{\alpha}^{p}\|B\|^{p}\left(\frac{t_{2}^{1-q \alpha}}{1-q \alpha}\right)^{\frac{p}{q}}\left(t_{2}-t_{1}\right)\left\|u^{\lambda}\right\|^{p} \\
& +6^{p-1} M_{\alpha}^{p} N_{\alpha}^{p}\|B\|^{p} E\left(\int_{0}^{t_{1}}\left(\frac{t_{1}-s}{2}\right)^{-2 \alpha} e^{-a\left(t_{1}-s\right)}\left(t_{2}-t_{1}\right)^{\alpha}\left\|u^{\lambda}(s)\right\| d s\right)^{p} \\
& \leq 6^{p-1} M_{\alpha}^{p}\|B\|^{p}\left(\frac{T^{1-q \alpha}}{1-q \alpha}\right)^{\frac{p}{q}}\left(t_{2}-t_{1}\right)\left\|u^{\lambda}\right\|^{p} \\
& +6^{p-1} M_{\alpha}^{p} N_{\alpha}^{p}\|B\|^{p}\left(t_{2}-t_{1}\right)^{p \alpha}\left(\int_{0}^{t_{1}}\left(\frac{t_{1}-s}{2}\right)^{-2 q \alpha} e^{-a q\left(t_{1}-s\right)} d s\right)^{\frac{p}{q}} E \int_{0}^{t_{1}}\left\|u^{\lambda}(s)\right\|^{p} d s \\
& \leq 6^{p-1} M_{\alpha}^{p}\|B\|^{p}\left(\frac{T^{1-q \alpha}}{1-q \alpha}\right)^{\frac{p}{q}}\left(t_{2}-t_{1}\right)\left\|u^{\lambda}\right\|^{p} \\
& +6^{p-1} M_{\alpha}^{p} N_{\alpha}^{p}\|B\|^{p}\left(t_{2}-t_{1}\right)^{p \alpha}\left(\int_{0}^{t_{1}}\left(\frac{t_{1}-s}{2}\right)^{-2 q \alpha} e^{-a q\left(t_{1}-s\right)} d s\right)^{\frac{p}{q}} E \int_{0}^{t_{1}}\left\|u^{\lambda}(s)\right\|^{p} d s \\
& \leq 6^{p-1} M_{\alpha}^{p}\|B\|^{p}\left(\frac{T^{1-q \alpha}}{1-q \alpha}\right)^{\frac{p}{q}}\left(t_{2}-t_{1}\right)\left\|u^{\lambda}\right\|^{p} \\
& +6^{p-1} M_{\alpha}^{p} N_{\alpha}^{p}\|B\|^{p}\left(t_{2}-t_{1}\right)^{p \alpha}\left(\frac{2^{q \alpha} T^{1-q \alpha}}{1-2 q \alpha}\right)^{\frac{p}{q}} T\left\|u^{\lambda}\right\|^{p} d s, \\
& I_{32}=3^{p-1} E \mid \int_{0}^{t_{2}} A^{\alpha} S\left(t_{2}-s\right) f\left(s, A^{-\alpha}\left(Y_{s}+\bar{\xi}_{s}\right), u(s)\right) d s \\
& -\left.\int_{0}^{t_{1}} A^{\alpha} S\left(t_{1}-s\right) f\left(s, A^{-\alpha}\left(Y_{s}+\bar{\xi}_{s}\right), u(s)\right) d s\right|^{p} \\
& \leq 6^{p-1} E\left|\int_{t_{1}}^{t_{2}} A^{\alpha} S\left(t_{2}-s\right) f\left(s, A^{-\alpha}\left(Y_{s}+\bar{\xi}_{s}\right), u(s)\right)\right|^{p} \\
& +6^{p-1} E\left|\int_{0}^{t_{1}} A^{\alpha}\left(S\left(t_{2}-s\right)-S\left(t_{1}-s\right)\right) f\left(s, A^{-\alpha}\left(Y_{s}+\bar{\xi}_{s}\right), u(s)\right) d s\right|^{p} \\
& \leq 6^{p-1} M_{\alpha}^{p}\left(\frac{T^{1-q \alpha}}{1-q \alpha}\right)^{\frac{p}{q}} \int_{t_{1}}^{t_{2}} E\left|f\left(s, A^{-\alpha}\left(Y_{s}+\bar{\xi}_{s}\right), u(s)\right)\right|^{p} d s \\
& +6^{p-1} M_{\alpha}^{p} N_{\alpha}^{p}\left(t_{2}-t_{1}\right)^{p \alpha}\left(\frac{2^{q \alpha} T^{1-q \alpha}}{1-2 q \alpha}\right)^{\frac{p}{q}} \int_{0}^{t_{1}} E\left|f\left(s, A^{-\alpha}\left(Y_{s}+\bar{\xi}_{s}\right), u(s)\right)\right|^{p} d s \\
& \leq 6^{p-1} M_{\alpha}^{p}\left(\frac{T^{1-q \alpha}}{1-q \alpha}\right)^{\frac{p}{q}}\left(t_{2}-t_{1}\right)
\end{aligned}
$$




$$
\begin{aligned}
& \times K_{1}\left(1+4^{p-1}\left(E\|\xi\|_{B_{h}^{\alpha}}^{p}+l^{p}\|Y\|_{T}^{p}+l^{p} M^{p} E\left|A^{\alpha} \xi(0)\right|^{p}\right)+\|u\|^{p}\right) \\
& +6^{p-1} M_{\alpha}^{p} N_{\alpha}^{p}\left(t_{2}-t_{1}\right)^{p \alpha}\left(\frac{2^{q \alpha} T^{1-q \alpha}}{1-2 q \alpha}\right)^{\frac{p}{q}} \\
& \times T K_{1}\left(1+4^{p-1}\left(E\|\xi\|_{B_{h}^{\alpha}}^{p}+l^{p}\|Y\|_{T}^{p}+l^{p} M^{p} E\left|A^{\alpha} \xi(0)\right|^{p}\right)+\|u\|^{p}\right), \\
& I_{33}=3^{p-1} E \mid \int_{0}^{t_{2}} A^{\alpha} S\left(t_{2}-s\right) g\left(s, A^{-\alpha}\left(Y_{s}+\bar{\xi}_{s}\right), u(s)\right) d w(s) \\
& -\left.\int_{0}^{t_{1}} A^{\alpha} S\left(t_{1}-s\right) g\left(s, A^{-\alpha}\left(Y_{s}+\bar{\xi}_{s}\right), u(s)\right) d w(s)\right|^{p} \\
& \leq 6^{p-1} E\left|\int_{t_{1}}^{t_{2}} A^{\alpha} S\left(t_{2}-s\right) g\left(s, A^{-\alpha}\left(Y_{s}+\bar{\xi}_{s}\right), u(s)\right) d w(s)\right|^{p} \\
& +6^{p-1} E\left|\int_{0}^{t_{1}} A^{\alpha}\left(S\left(t_{2}-s\right)-S\left(t_{1}-s\right)\right) g\left(s, A^{-\alpha}\left(Y_{s}+\bar{\xi}_{s}\right), u(s)\right) d w(s)\right|^{p} \\
& \leq 6^{p-1} C_{p} E\left(\int_{t_{1}}^{t_{2}}\left\|A^{\alpha} S\left(t_{2}-s\right) g\left(s, A^{-\alpha}\left(Y_{s}+\bar{\xi}_{s}\right), u(s)\right)\right\|_{2}^{2} d s\right)^{\frac{p}{2}} \\
& +6^{p-1} C_{p} E\left(\int_{0}^{t_{1}}\left\|A^{\alpha}\left(S\left(t_{2}-s\right)-S\left(t_{1}-s\right)\right) g\left(s, A^{-\alpha}\left(Y_{s}+\bar{\xi}_{s}\right), u(s)\right)\right\|_{2}^{2} d s\right)^{\frac{p}{2}} \\
& \leq 6^{p-1} C_{p} M_{\alpha}^{p} E\left(\int_{t_{1}}^{t_{2}}\left(t_{2}-s\right)^{-2 \alpha} e^{-2 a\left(t_{2}-s\right)}\left\|g\left(s, A^{-\alpha}\left(Y_{s}+\bar{\xi}_{s}\right), u(s)\right)\right\|_{2}^{2} d s\right)^{\frac{p}{2}} \\
& +6^{p-1} C_{p} E\left(\int_{0}^{t_{1}} \| A^{\alpha} S\left(\frac{t_{1}-s}{2}\right)\left(S\left(t_{2}-t_{1}\right)-I\right)\right. \\
& \left.\times S\left(\frac{t_{1}-s}{2}\right) g\left(s, A^{-\alpha}\left(Y_{s}+\bar{\xi}_{s}\right), u(s)\right) \|_{2}^{2} d s\right)^{\frac{p}{2}} \\
& \leq 6^{p-1} C_{p} M_{\alpha}^{p}\left(\int_{t_{1}}^{t_{2}}\left(\left(t_{2}-s\right)^{-2 \alpha} e^{-2 a\left(t_{2}-s\right)}\right)^{\frac{p}{p-2}} d s\right)^{\frac{p-2}{p}} \\
& \times \int_{t_{1}}^{t_{2}} E\left\|g\left(s, A^{-\alpha}\left(Y_{s}+\bar{\xi}_{s}\right), u(s)\right)\right\|_{2}^{p} d s \\
& +6^{p-1} C_{p} M_{\alpha}^{p} N_{\alpha}^{p} E\left(\int_{0}^{t_{1}}\left(\frac{t_{2}-s}{2}\right)^{-2 \alpha} e^{-a\left(t_{2}-s\right)}\left(t_{2}-t_{1}\right)^{\alpha}\right. \\
& \left.\times\left\|g\left(s, A^{-\alpha}\left(Y_{s}+\bar{\xi}_{s}\right), u(s)\right)\right\|_{2}^{2} d s\right)^{\frac{p}{2}} \\
& \leq 6^{p-1} C_{p} M_{\alpha}^{p}\left(\frac{T^{1-\frac{2 \alpha p}{p-2}}}{1-\frac{2 \alpha p}{p-2}}\right)^{\frac{p-2}{p}} \int_{t_{1}}^{t_{2}} E\left\|g\left(s, A^{-\alpha}\left(Y_{s}+\bar{\xi}_{s}\right), u(s)\right)\right\|_{2}^{p} d s \\
& +6^{p-1} C_{p} M_{\alpha}^{p} N_{\alpha}^{p}\left(t_{2}-t_{1}\right)^{p \alpha} 2^{\frac{p-2}{p}}\left(\frac{\left(\frac{T}{2}\right)^{1-\frac{2 \alpha p}{p-2}}}{1-\frac{2 \alpha p}{p-2}}\right)^{\frac{p-2}{p}} \int_{0}^{t_{1}} E\left\|g\left(s, A^{-\alpha}\left(Y_{s}+\bar{\xi}_{s}\right), u(s)\right)\right\|_{2}^{p} d s \\
& \leq 6^{p-1} C_{p} M_{\alpha}^{p}\left(\frac{T^{1-\frac{2 \alpha p}{p-2}}}{1-\frac{2 \alpha p}{p-2}}\right)^{\frac{p-2}{p}}\left(t_{1}-t_{2}\right) \\
& \times K_{1}\left(1+4^{p-1}\left(E\|\xi\|_{B_{h}^{\alpha}}^{p}+l^{p}\|Y\|_{T}^{p}+l^{p} M^{p} E\left|A^{\alpha} \xi(0)\right|^{p}\right)+\|u\|^{p}\right)
\end{aligned}
$$




$$
\begin{aligned}
& +6^{p-1} C_{p} M_{\alpha}^{p} N_{\alpha}^{p}\left(t_{2}-t_{1}\right)^{p \alpha} 2^{\frac{p-2}{p}}\left(\frac{\left(\frac{T}{2}\right)^{1-\frac{2 \alpha p}{p-2}}}{1-\frac{2 \alpha p}{p-2}}\right)^{\frac{p-2}{p}} \\
& \times T K_{1}\left(1+4^{p-1}\left(E\|\xi\|_{B_{h}^{\alpha}}^{p}+l^{p}\|Y\|_{T}^{p}+l^{p} M^{p} E\left|A^{\alpha} \xi(0)\right|^{p}\right)+\|u\|^{p}\right) .
\end{aligned}
$$

Then it is clear that $E\left|Y^{\lambda}\left(t_{2}\right)-Y^{\lambda}\left(t_{1}\right)\right|^{p} \rightarrow 0$ as $t_{2} \rightarrow t_{1}$, therefore $Y^{\lambda}(t)$ is continuous in $L^{p}$ sense.

From all inequalities above, we conclude that the operator $\Phi_{\lambda}$ maps $B_{T}^{0} \times C\left(J, L^{P}(\Omega\right.$, $\Im, P ; U))$ into $B_{T}^{0} \times C\left(J, L^{P}(\Omega, \Im, P ; U)\right)$. The proof is completed.

Lemma 3.6 Let $0<\alpha<\frac{p-2}{2 p}$, and $\left(\mathrm{A}_{1}\right),\left(\mathrm{A}_{2}\right)$ and $L^{p}$ with $p$ lower case hold, then for any $\lambda>0$, the operator $\Phi_{\lambda}$ has a unique fixed point in $B_{T}^{0} \times C\left(J, L^{P}(\Omega, \Im, P ; U)\right)$.

Proof The proof is based on the Banach fixed point theorem for contractions. In the proof, we take $\Phi_{\lambda}\left(Y_{1}, u_{1}\right)(t)=\left(Y_{1}^{\lambda}(t), u_{1}^{\lambda}(t)\right)$ and $\Phi_{\lambda}\left(Y_{2}, u_{2}\right)(t)=\left(Y_{2}^{\lambda}(t), u_{2}^{\lambda}(t)\right)$. Then we have

$$
\begin{aligned}
& E\left\|u_{2}^{\lambda}(t)-u_{1}^{\lambda}(t)\right\|^{p} \\
& =E \| B^{*} S^{*}(T-t) \int_{0}^{t}\left(\lambda I+\Gamma_{s}^{T}\right)^{-1} S(T-s)\left(f\left(s, A^{-\alpha}\left(Y_{2 s}+\bar{\xi}_{s}\right), u_{2}(s)\right)\right. \\
& \left.-f\left(s, A^{-\alpha}\left(Y_{1 s}+\bar{\xi}_{s}\right), u_{1}(s)\right)\right) d s \\
& +B^{*} S^{*}(T-t) \int_{0}^{t}\left(\lambda I+\Gamma_{s}^{T}\right)^{-1} S(T-s)\left(g\left(s, A^{-\alpha}\left(Y_{2 s}+\bar{\xi}_{s}\right), u_{2}(s)\right)\right. \\
& \left.-g\left(s, A^{-\alpha}\left(Y_{1 s}+\bar{\xi}_{s}\right), u_{1}(s)\right)\right) d w(s) \|^{p} \\
& \leq 2^{p-1} E \| B^{*} S^{*}(T-t) \int_{0}^{t}\left(\lambda I+\Gamma_{s}^{T}\right)^{-1} S(T-s)\left(f\left(s, A^{-\alpha}\left(Y_{2 s}+\bar{\xi}_{s}\right), u_{2}(s)\right)\right. \\
& \left.-f\left(s, A^{-\alpha}\left(Y_{1 s}+\bar{\xi}_{s}\right), u_{1}(s)\right)\right) d s \|^{p} \\
& +2^{p-1} E \| B^{*} S^{*}(T-t) \int_{0}^{t}\left(\lambda I+\Gamma_{s}^{T}\right)^{-1} S(T-s)\left(g\left(s, A^{-\alpha}\left(Y_{2 s}+\bar{\xi}_{s}\right), u_{2}(s)\right)\right. \\
& \left.-g\left(s, A^{-\alpha}\left(Y_{1 s}+\bar{\xi}_{s}\right), u_{1}(s)\right)\right) d w(s) \|^{p} \\
& \leq 2^{p-1}\|B\|^{p} M^{2 p} \frac{1}{\lambda^{p}} T^{p-1} E \int_{0}^{t} \mid f\left(s, A^{-\alpha}\left(Y_{2 s}+\bar{\xi}_{s}\right), u_{2}(s)\right) \\
& -\left.f\left(s, A^{-\alpha}\left(Y_{1 s}+\bar{\xi}_{s}\right), u_{1}(s)\right)\right|^{p} d s \\
& +2^{p-1}\|B\|^{p} M^{2 p} \frac{1}{\lambda^{p}} T^{\frac{p}{2}-1} E \int_{0}^{t} \| g\left(s, A^{-\alpha}\left(Y_{2 s}+\bar{\xi}_{s}\right), u_{2}(s)\right) \\
& -g\left(s, A^{-\alpha}\left(Y_{1 s}+\bar{\xi}_{s}\right), u_{1}(s)\right) \|_{2}^{p} d s \\
& \leq 2^{p-1}\|B\|^{p} M^{2 p} \frac{1}{\lambda^{p}} T^{p-1} K_{1} \int_{0}^{t}\left(E\left\|A^{-\alpha}\left(Y_{2 s}+\bar{\xi}_{s}\right)-A^{-\alpha}\left(Y_{1 s}+\bar{\xi}_{s}\right)\right\|_{B_{h}^{\alpha}}^{p}\right. \\
& \left.+E\left\|u_{2}(s)-u_{1}(s)\right\|^{p}\right) d s+2^{p-1}\|B\|^{p} M^{2 p} \frac{1}{\lambda^{p}} T^{\frac{p}{2}-1} \\
& \times K_{1} \int_{0}^{t}\left(E\left\|A^{-\alpha}\left(Y_{2 s}+\bar{\xi}_{s}\right)-A^{-\alpha}\left(Y_{1 s}+\bar{\xi}_{s}\right)\right\|_{B_{h}^{\alpha}}^{p}+E\left\|u_{2}(s)-u_{1}(s)\right\|^{p}\right) d s
\end{aligned}
$$




$$
\begin{aligned}
\leq & 2^{p-1}\|B\|^{p} M^{2 p} \frac{1}{\lambda^{p}}\left(T^{p-1}+T^{\frac{p}{2}-1}\right) K_{1} \int_{0}^{t} E\left\|Y_{2 s}-Y_{1 s}\right\|_{B_{h}}^{p} d s \\
& +2^{p-1}\|B\|^{p} M^{2 p} \frac{1}{\lambda^{p}}\left(T^{p-1}+T^{\frac{p}{2}-1}\right) K_{1} \int_{0}^{t} E\left\|u_{2}(s)-u_{1}(s)\right\|^{p} d s \\
\leq & 4^{p-1}\|B\|^{p} M^{2 p} \frac{1}{\lambda^{p}}\left(T^{p-1}+T^{\frac{p}{2}-1}\right) K_{1} l^{p} \int_{0}^{t} E \sup _{0 \leq \tau \leq s}\left|Y_{2}(\tau)-Y_{1}(\tau)\right|^{p} d s \\
& +2^{p-1}\|B\|^{p} M^{2 p} \frac{1}{\lambda^{p}}\left(T^{p-1}+T^{\frac{p}{2}-1}\right) K_{1} \int_{0}^{t} E\left\|u_{2}(s)-u_{1}(s)\right\|^{p} d s,
\end{aligned}
$$

which yields that there exists a constant $K_{3}$ such that

$$
\begin{aligned}
E \| & u_{2}^{\lambda}(t)-u_{1}^{\lambda}(t) \|^{p} \\
& \leq K_{3}\left(\int_{0}^{t} E\left\|u_{2}(s)-u_{1}(s)\right\|^{p} d s+\int_{0}^{t} E \sup _{0 \leq \tau \leq s}\left|Y_{2}(\tau)-Y_{1}(\tau)\right|^{p} d s\right) \\
& =K_{3} \int_{0}^{t}\left(E\left\|u_{2}(s)-u_{1}(s)\right\|^{p}+E \sup _{0 \leq \tau \leq s}\left|Y_{2}(\tau)-Y_{1}(\tau)\right|^{p}\right) d s .
\end{aligned}
$$

For $Y_{2}^{\lambda}$ and $Y_{1}^{\lambda}$, we have

$$
\begin{aligned}
E \sup _{0 \leq s \leq t}\left|Y_{2}^{\lambda}(s)-Y_{1}^{\lambda}(s)\right|^{p} \\
=E \sup _{0 \leq s \leq t} \mid \int_{0}^{s} A^{\alpha} S(s-\tau) B\left(u_{2}^{\lambda}(\tau)-u_{1}^{\lambda}(\tau)\right) d \tau \\
\quad+\int_{0}^{s} A^{\alpha} S(s-\tau)\left(f\left(\tau, A^{-\alpha}\left(Y_{2 \tau}+\bar{\xi}_{\tau}\right), u_{2}(\tau)\right)-f\left(\tau, A^{-\alpha}\left(Y_{1 \tau}+\bar{\xi}_{\tau}\right), u_{1}(\tau)\right)\right) d \tau \\
\quad+\left.\int_{0}^{s} A^{\alpha} S(s-\tau)\left(g\left(\tau, A^{-\alpha}\left(Y_{2 \tau}+\bar{\xi}_{\tau}\right), u_{2}(\tau)\right)-g\left(\tau, A^{-\alpha}\left(Y_{1 \tau}+\bar{\xi}_{\tau}\right), u_{1}(\tau)\right)\right) d w(\tau)\right|^{p} \\
\leq 3^{p-1} E \sup _{0 \leq s \leq t}\left|\int_{0}^{s} A^{\alpha} S(s-\tau) B\left(u_{2}^{\lambda}(\tau)-u_{1}^{\lambda}(\tau)\right) d \tau\right|^{p} \\
\quad+3^{p-1} E \sup _{0 \leq s \leq t} \mid \int_{0}^{s} A^{\alpha} S(s-\tau)\left(f\left(\tau, A^{-\alpha}\left(Y_{2 \tau}+\bar{\xi}_{\tau}\right), u_{2}(\tau)\right)\right. \\
\left.\quad-f\left(\tau, A^{-\alpha}\left(Y_{1 \tau}+\bar{\xi}_{\tau}\right), u_{1}(\tau)\right)\right)\left.d \tau\right|^{p} \\
\quad+3^{p-1} E \sup _{0 \leq s \leq t} \mid \int_{0}^{s} A^{\alpha} S(s-\tau)\left(g\left(\tau, A^{-\alpha}\left(Y_{2 \tau}+\bar{\xi}_{\tau}\right), u_{2}(\tau)\right)\right. \\
\left.\quad-g\left(\tau, A^{-\alpha}\left(Y_{1 \tau}+\bar{\xi}_{\tau}\right), u_{1}(\tau)\right)\right)\left.d w(\tau)\right|^{p} \\
=I_{41}+I_{42}+I_{43},
\end{aligned}
$$

and

$$
\begin{aligned}
I_{41} & =3^{p-1} E \sup _{0 \leq s \leq t}\left|\int_{0}^{s} A^{\alpha} S(s-\tau) B\left(u_{2}^{\lambda}(\tau)-u_{1}^{\lambda}(\tau)\right) d \tau\right|^{p} \\
& \leq 3^{p-1} E \sup _{0 \leq s \leq t}\left(\int_{0}^{s}\left|A^{\alpha} S(s-\tau) B\left(u_{2}^{\lambda}(\tau)-u_{1}^{\lambda}(\tau)\right)\right| d \tau\right)^{p}
\end{aligned}
$$




$$
\begin{aligned}
& \leq 3^{p-1} E \sup _{0 \leq s \leq t}\left(\int_{0}^{s} M_{\alpha} e^{-a(s-\tau)}(s-\tau)^{-\alpha}\left|B\left(u_{2}^{\lambda}(\tau)-u_{1}^{\lambda}(\tau)\right)\right| d \tau\right)^{p} \\
& \leq 3^{p-1}\|B\|^{p} M_{\alpha}^{p} E \sup _{0 \leq s \leq t}\left[\left(\int_{0}^{s}(s-\tau)^{-\alpha q} d \tau\right)^{\frac{p}{q}} \int_{0}^{s}\left\|u_{2}^{\lambda}(\tau)-u_{1}^{\lambda}(\tau)\right\|^{p} d \tau\right] \\
& =3^{p-1}\|B\|^{p} M_{\alpha}^{p} E \sup _{0 \leq s \leq t}\left[\left(\frac{s^{1-\alpha q}}{1-\alpha q}\right)^{\frac{p}{q}} \int_{0}^{s}\left\|u_{2}^{\lambda}(\tau)-u_{1}^{\lambda}(\tau)\right\|^{p} d \tau\right] \\
& =3^{p-1}\|B\|^{p} M_{\alpha}^{p}\left(\frac{t^{1-\alpha q}}{1-\alpha q}\right)^{\frac{p}{q}} E \int_{0}^{t}\left\|u_{2}^{\lambda}(s)-u_{1}^{\lambda}(s)\right\|^{p} d s \\
& \leq 3^{p-1}\|B\|^{p} M_{\alpha}^{p}\left(\frac{T^{1-\alpha q}}{1-\alpha q}\right)^{\frac{p}{q}} T \sup _{0 \leq s \leq t} E\left\|u_{2}^{\lambda}(s)-u_{1}^{\lambda}(s)\right\|^{p} \\
& \leq 3^{p-1}\|B\|^{p} M_{\alpha}^{p}\left(\frac{T^{1-\alpha q}}{1-\alpha q}\right)^{\frac{p}{q}} T \sup _{0 \leq s \leq t} K_{3} \int_{0}^{s}\left(E\left\|u_{2}(\tau)-u_{1}(\tau)\right\|^{p}\right. \\
& \left.+E \sup _{0 \leq \sigma \leq \tau}\left|Y_{2}(\sigma)-Y_{1}(\sigma)\right|^{p}\right) d \tau \\
& \leq 3^{p-1}\|B\|^{p} M_{\alpha}^{p}\left(\frac{T^{1-\alpha q}}{1-\alpha q}\right)^{\frac{p}{q}} T K_{3} \int_{0}^{t}\left(E\left\|u_{2}(s)-u_{1}(s)\right\|^{p}\right. \\
& \left.+E \sup _{0 \leq \tau \leq s}\left|Y_{2}(\tau)-Y_{1}(\tau)\right|^{p}\right) d s, \\
& I_{42}=3^{p-1} E \sup _{0 \leq s \leq t} \mid \int_{0}^{s} A^{\alpha} S(s-\tau)\left(f\left(\tau, A^{-\alpha}\left(Y_{2 \tau}+\bar{\xi}_{\tau}\right), u_{2}(\tau)\right)\right. \\
& \left.-f\left(\tau, A^{-\alpha}\left(Y_{1 \tau}+\bar{\xi}_{\tau}\right), u_{1}(\tau)\right)\right)\left.d \tau\right|^{p} \\
& \leq 3^{p-1} E \sup _{0 \leq s \leq t}\left(\int_{0}^{s} \mid A^{\alpha} S(s-\tau)\left(f\left(\tau, A^{-\alpha}\left(Y_{2 \tau}+\bar{\xi}_{\tau}\right), u_{2}(\tau)\right)\right.\right. \\
& \left.\left.-f\left(\tau, A^{-\alpha}\left(Y_{1 \tau}+\bar{\xi}_{\tau}\right), u_{1}(\tau)\right)\right) \mid d \tau\right)^{p} \\
& \leq 3^{p-1} E \sup _{0 \leq s \leq t}\left(\int_{0}^{s} M_{\alpha} e^{-a(s-r)}(s-r)^{-\alpha} \mid f\left(\tau, A^{-\alpha}\left(Y_{2 \tau}+\bar{\xi}_{\tau}\right), u_{2}(\tau)\right)\right. \\
& \left.-f\left(\tau, A^{-\alpha}\left(Y_{1 \tau}+\bar{\xi}_{\tau}\right), u_{1}(\tau)\right) \mid d \tau\right)^{p} \\
& \leq 3^{p-1}\|B\|^{p} M_{\alpha}^{p} E \sup _{0 \leq s \leq t}\left[\left(\int_{0}^{s}(s-r)^{-\alpha q} d s\right)^{\frac{p}{q}} \int_{0}^{s} \mid f\left(\tau, A^{-\alpha}\left(Y_{2 \tau}+\bar{\xi}_{\tau}\right), u_{2}(\tau)\right)\right. \\
& \left.-\left.f\left(\tau, A^{-\alpha}\left(Y_{1 \tau}+\bar{\xi}_{\tau}\right), u_{1}(\tau)\right)\right|^{p} d \tau\right] \\
& =3^{p-1}\|B\|^{p} M_{\alpha}^{p} E \sup _{0 \leq s \leq t}\left(\frac{s^{1-\alpha q}}{1-\alpha q}\right)^{\frac{p}{q}} \int_{0}^{s} \mid f\left(\tau, A^{-\alpha}\left(Y_{2 \tau}+\bar{\xi}_{\tau}\right), u_{2}(\tau)\right) \\
& -\left.f\left(\tau, A^{-\alpha}\left(Y_{1 \tau}+\bar{\xi}_{\tau}\right), u_{1}(\tau)\right)\right|^{p} d \tau \\
& =3^{p-1}\|B\|^{p} M_{\alpha}^{p}\left(\frac{t^{1-\alpha q}}{1-\alpha q}\right)^{\frac{p}{q}} E \int_{0}^{t} \mid f\left(s, A^{-\alpha}\left(Y_{2 s}+\bar{\xi}_{s}\right), u_{2}(s)\right) \\
& -\left.f\left(s, A^{-\alpha}\left(Y_{1 s}+\bar{\xi}_{s}\right), u_{1}(s)\right)\right|^{p} d s
\end{aligned}
$$




$$
\begin{aligned}
& \leq 3^{p-1}\|B\|^{p} M_{\alpha}^{p}\left(\frac{T^{1-\alpha q}}{1-\alpha q}\right)^{\frac{p}{q}} K_{1} \int_{0}^{t}\left(E \| A^{-\alpha}\left(Y_{2 s}+\bar{\xi}_{s}\right)\right. \\
& \left.-A^{-\alpha}\left(Y_{1 s}+\bar{\xi}_{s}\right)\left\|_{B_{h}^{\alpha}}^{p}+E\right\| u_{2}(s)-u_{1}(s) \|^{p}\right) d s \\
& =3^{p-1}\|B\|^{p} M_{\alpha}^{p}\left(\frac{T^{1-\alpha q}}{1-\alpha q}\right)^{\frac{p}{q}} K_{1} \int_{0}^{t}\left(E\left\|Y_{2 s}-Y_{1 s}\right\|_{B_{h}}^{p}+E\left\|u_{2}(s)-u_{1}(s)\right\|^{p}\right) d s \\
& \leq 3^{p-1}\|B\|^{p} M_{\alpha}^{p}\left(\frac{T^{1-\alpha q}}{1-\alpha q}\right)^{\frac{p}{q}} K_{1} \int_{0}^{t}\left(2^{p-1} l^{p} E \sup _{0 \leq \tau \leq s}\left|Y_{2}(\tau)-Y_{1}(\tau)\right|^{p}\right. \\
& \left.+E\left\|u_{2}(s)-u_{1}(s)\right\|^{p}\right) d s \\
& \leq 6^{p-1}\|B\|^{p} M_{\alpha}^{p}\left(\frac{T^{1-\alpha q}}{1-\alpha q}\right)^{\frac{p}{q}} K_{1} l^{p} \int_{0}^{t} E \sup _{0 \leq \tau \leq s}\left|Y_{2}(\tau)-Y_{1}(\tau)\right|^{p} d s \\
& +3^{p-1}\|B\|^{p} M_{\alpha}^{p}\left(\frac{T^{1-\alpha q}}{1-\alpha q}\right)^{\frac{p}{q}} K_{1} \int_{0}^{t} E\left\|u_{2}(s)-u_{1}(s)\right\|^{p} d s, \\
& I_{43}=3^{p-1} E \sup _{0 \leq s \leq t} \mid \int_{0}^{s} A^{\alpha} S(s-\tau)\left(g\left(\tau, A^{-\alpha}\left(Y_{2 \tau}+\bar{\xi}_{\tau}\right), u_{2}(\tau)\right)\right. \\
& \left.-g\left(\tau, A^{-\alpha}\left(Y_{1 \tau}+\bar{\xi}_{\tau}\right), u_{1}(\tau)\right)\right)\left.d w(\tau)\right|^{p} \\
& \leq 3^{p-1} C_{p} E\left(\int_{0}^{t} \| A^{\alpha} S(t-s)\left(g\left(s, A^{-\alpha}\left(Y_{2 s}+\bar{\xi}_{s}\right), u_{2}(s)\right)\right.\right. \\
& \left.\left.-g\left(s, A^{-\alpha}\left(Y_{1 s}+\bar{\xi}_{s}\right), u_{1}(s)\right)\right) \|_{2}^{2} d s\right)^{\frac{p}{2}} \\
& \leq 3^{p-1} C_{p} E\left(\int_{0}^{t} M_{\alpha}^{2} e^{-2 a(t-s)}(t-s)^{-2 \alpha} \| g\left(s, A^{-\alpha}\left(Y_{2 s}+\bar{\xi}_{s}\right), u_{2}(s)\right)\right. \\
& \left.-g\left(s, A^{-\alpha}\left(Y_{1 s}+\bar{\xi}_{s}\right), u_{1}(s)\right) \|_{2}^{2} d s\right)^{\frac{p}{2}} \\
& \leq 3^{p-1} C_{p} M_{\alpha}^{p}\left(\frac{t^{1-\frac{2 \alpha p}{p-2}}}{1-\frac{2 \alpha p}{p-2}}\right)^{\frac{p-2}{2}} \int_{0}^{t} E \| g\left(s, A^{-\alpha}\left(Y_{2 s}+\bar{\xi}_{s}\right), u_{2}(s)\right) \\
& -g\left(s, A^{-\alpha}\left(Y_{1 s}+\bar{\xi}_{s}\right), u_{1}(s)\right) \|_{2}^{p} d s \\
& \leq 3^{p-1} C_{p} M_{\alpha}^{p}\left(\frac{T^{1-\frac{2 \alpha p}{p-2}}}{1-\frac{2 \alpha p}{p-2}}\right)^{\frac{p-2}{2}} K_{1} \int_{0}^{t}\left(E\left\|A^{-\alpha}\left(Y_{2 s}+\bar{\xi}_{s}\right)-A^{-\alpha}\left(Y_{1 s}+\bar{\xi}_{s}\right)\right\|_{B_{h}^{\alpha}}^{p}\right. \\
& \left.+E\left\|u_{2}(s)-u_{1}(s)\right\|^{p}\right) d s \\
& =3^{p-1} C_{p} M_{\alpha}^{p}\left(\frac{T^{1-\frac{2 \alpha p}{p-2}}}{1-\frac{2 \alpha p}{p-2}}\right)^{\frac{p-2}{2}} K_{1} \int_{0}^{t}\left(E\left\|Y_{2 s}-Y_{1 s}\right\|_{B_{h}}^{p}+E\left\|u_{2}(s)-u_{1}(s)\right\|^{p}\right) d s \\
& \leq 3^{p-1} C_{p} M_{\alpha}^{p}\left(\frac{T^{1-\frac{2 \alpha p}{p-2}}}{1-\frac{2 \alpha p}{p-2}}\right)^{\frac{p-2}{2}} K_{1} \int_{0}^{t}\left(2^{p-1} l^{p} E \sup _{0 \leq \tau \leq s}\left|Y_{2}(\tau)-Y_{1}(\tau)\right|^{p}\right. \\
& \left.+E\left\|u_{2}(s)-u_{1}(s)\right\|^{p}\right) d s
\end{aligned}
$$




$$
\begin{aligned}
\leq & 6^{p-1} C_{p} M_{\alpha}^{p}\left(\frac{T^{1-\frac{2 \alpha p}{p-2}}}{1-\frac{2 \alpha p}{p-2}}\right)^{\frac{p-2}{2}} K_{1} l^{p} \int_{0}^{t} E \sup _{0 \leq \tau \leq s}\left|Y_{2}(\tau)-Y_{1}(\tau)\right|^{p} d s \\
& +3^{p-1} C_{p} M_{\alpha}^{p}\left(\frac{T^{1-\frac{2 \alpha p}{p-2}}}{1-\frac{2 \alpha p}{p-2}}\right)^{\frac{p-2}{2}} K_{1} \int_{0}^{t} E\left\|u_{2}(s)-u_{1}(s)\right\|^{p} d s .
\end{aligned}
$$

From the estimates $I_{41}, I_{42}$ and $I_{43}$, we deduce that there exists a constant $K_{4}$ such that

$$
\begin{aligned}
E & \sup _{0 \leq s \leq t}\left|Y_{2}^{\lambda}(s)-Y_{1}^{\lambda}(s)\right|^{p} \\
& \leq K_{4}\left(\int_{0}^{t} E \sup _{0 \leq \tau \leq s}\left|Y_{2}(\tau)-Y_{1}(\tau)\right|^{p} d s+\int_{0}^{t} E\left\|u_{2}(s)-u_{1}(s)\right\|^{p} d s\right) \\
& =K_{4} \int_{0}^{t}\left(E \sup _{0 \leq \tau \leq s}\left|Y_{2}(\tau)-Y_{1}(\tau)\right|^{p}+E\left\|u_{2}(s)-u_{1}(s)\right\|^{p}\right) d s .
\end{aligned}
$$

Denote $K_{5}=K_{3}+K_{4}$. It can be derived that

$$
\begin{aligned}
& E \sup _{0 \leq s \leq t}\left|Y_{2}^{\lambda}(s)-Y_{1}^{\lambda}(s)\right|^{p}+E\left\|u_{2}^{\lambda}(t)-u_{1}^{\lambda}(t)\right\|^{p} \\
& \quad \leq K_{5} \int_{0}^{t}\left(E \sup _{0 \leq \tau \leq s}\left|Y_{2}(\tau)-Y_{1}(\tau)\right|^{p}+E\left\|u_{2}(s)-u_{1}(s)\right\|^{p}\right) d s \\
& \quad \leq K_{5} t\left(\left\|Y_{2}-Y_{1}\right\|_{T}^{p}+\left\|u_{2}-u_{1}\right\|^{p}\right) .
\end{aligned}
$$

We denote that for any $(Y, u) \in B_{T}^{0} \times C\left(J, L^{p}(\Omega, \Im, P, U)\right), \Phi_{\lambda}^{2}(Y, u)=\Phi_{\lambda} \Phi_{\lambda}(Y, u)=$ $\Phi_{\lambda}\left(Y^{\lambda}, u^{\lambda}\right)=:\left(Y^{2 \lambda}, u^{2 \lambda}\right)$. Similarly, noting $\Phi_{\lambda}^{n}(Y, u)=\left(Y^{n \lambda}, u^{n \lambda}\right)$, we can obtain that for $\Phi_{\lambda}^{n}\left(Y_{1}, u_{1}\right)=\left(Y_{1}^{n \lambda}, u_{1}^{n \lambda}\right)$ and $\Phi_{\lambda}^{n}\left(Y_{2}, u_{2}\right)=\left(Y_{2}^{n \lambda}, u_{2}^{n \lambda}\right), n=1,2, \ldots$,

$$
\begin{aligned}
E & \sup _{0 \leq s \leq t}\left|Y_{2}^{2 \lambda}(s)-Y_{1}^{2 \lambda}(s)\right|^{p}+E\left\|u_{2}^{2 \lambda}(t)-u_{1}^{2 \lambda}(t)\right\|^{p} \\
& \leq K_{5} \int_{0}^{t}\left(E \sup _{0 \leq \tau \leq s}\left|Y_{2}^{\lambda}(\tau)-Y_{1}^{\lambda}(\tau)\right|^{p}+E\left\|u_{2}^{\lambda}(s)-u_{1}^{\lambda}(s)\right\|^{p}\right) d s \\
& \leq K_{5}^{2} \int_{0}^{t} \int_{0}^{s}\left(E \sup _{0 \leq \sigma \leq \tau}\left|Y_{2}(\sigma)-Y_{1}(\sigma)\right|^{p}+E\left\|u_{2}(\tau)-u_{1}(\tau)\right\|^{p}\right) d \tau d s \\
& \leq K_{5}^{2} \frac{t^{2}}{2 !}\left(\left\|Y_{2}-Y_{1}\right\|_{T}^{p}+\left\|u_{2}-u_{1}\right\|^{p}\right) .
\end{aligned}
$$

By a simple iteration, it can be obtained that for $n=1,2, \ldots$,

$$
\begin{aligned}
& E \sup _{0 \leq t \leq T}\left|Y_{2}^{n \lambda}(t)-Y_{1}^{n \lambda}(t)\right|^{p}+\sup _{0 \leq t \leq T} E\left\|u_{2}^{n \lambda}(t)-u_{1}^{n \lambda}(t)\right\|^{p} \\
& \leq K_{5}^{n} \frac{T^{n}}{n !}\left(\left\|Y_{2}-Y_{1}\right\|_{T}^{p}+\left\|u_{2}-u_{1}\right\|^{p}\right) .
\end{aligned}
$$

It is obvious that for some sufficiently large $n$, it holds that $2^{p-1} K_{5}^{n} \frac{T^{n}}{n !}<1$. In the following, let $L=2^{p-1} K_{5}^{n} \frac{T^{n}}{n !}<1$.

$$
\begin{aligned}
& \left\|\Phi_{\lambda}^{n}\left(Y_{1}, u_{1}\right)-\Phi_{\lambda}^{n}\left(Y_{2}, u_{2}\right)\right\|^{p} \\
& \quad=\left(\left(E \sup _{0 \leq t \leq T}\left|Y_{2}^{n \lambda}(t)-Y_{1}^{n \lambda}(t)\right|^{p}\right)^{\frac{1}{p}}+\sup _{0 \leq t \leq T}\left(E\left\|u_{2}^{n \lambda}(t)-u_{1}^{n \lambda}(t)\right\|^{p}\right)^{\frac{1}{p}}\right)^{p}
\end{aligned}
$$




$$
\begin{aligned}
& \leq 2^{p-1}\left(E \sup _{0 \leq t \leq T}\left|Y_{2}^{n \lambda}(t)-Y_{1}^{n \lambda}(t)\right|^{p}+\sup _{0 \leq t \leq T} E\left\|u_{2}^{n \lambda}(t)-u_{1}^{n \lambda}(t)\right\|^{p}\right) \\
& \leq L\left(\left\|Y_{2}-Y_{1}\right\|_{T}^{p}+\left\|u_{2}-u_{1}\right\|^{p}\right) \\
& \leq L\left(\left\|Y_{2}-Y_{1}\right\|_{T}+\left\|u_{2}-u_{1}\right\|\right)^{p},
\end{aligned}
$$

which implies

$$
\begin{aligned}
\left\|\Phi_{\lambda}^{n}\left(Y_{1}, u_{1}\right)-\Phi_{\lambda}^{n}\left(Y_{2}, u_{2}\right)\right\| & \leq L^{\frac{1}{p}}\left(\left\|Y_{2}-Y_{1}\right\|+\left\|u_{2}-u_{1}\right\|\right) \\
& <\left(\left\|Y_{2}-Y_{1}\right\|+\left\|u_{2}-u_{1}\right\|\right)=\left\|\left(Y_{2}, u_{2}\right)-\left(Y_{1}, u_{1}\right)\right\| .
\end{aligned}
$$

This shows that for large enough $n, \Phi_{\lambda}^{n}$ is a contraction. Thus, $\Phi_{\lambda}$ has a unique fixed point in $B_{T}^{0} \times C\left(J, L^{p}(\Omega, \Im, P, U)\right)$. We complete the proof.

Remark 3.7 The general Banach space $B_{T}^{0} \times C\left(J, L^{p}(\Omega, \Im, P ; U)\right)$ and the operator $\Phi_{\lambda}$ are constructed to transform the controllability problem into a fixed point problem. It shall be emphasized that the fundamental Lemma 3.4 is purposely established, which plays an important role in obtaining the boundedness of the operator and in solving the problems brought by the infinite delays. Although the control input and nonlinearity exist both in the drift and diffusion terms of the system, which makes it complicated to prove the contraction property of the operator, some inequality techniques are deliberately adopted as demonstrated in the proof of Lemma 3.6, which can effectively overcome the difficulty. With these techniques, the main conclusion will be established in a more general space with less restrictive conditions.

For any $\lambda>0$, let $\left(Y^{\lambda}, u^{\lambda}\right)$ be the fixed point of $\Phi_{\lambda}, Z^{\lambda}(t)=Y^{\lambda}(t)+\bar{\xi}(t)$ and $x^{\lambda}(t)=$ $A^{-\alpha} Z^{\lambda}(t)$. Then $\left(Z^{\lambda}(t), u^{\lambda}(t)\right)$ is the unique fixed point of the operator $\Psi_{\lambda}$, and $x^{\lambda}(t)$ is a solution of system (1). We also have

$$
\begin{aligned}
x^{\lambda}(t)= & S(t) \xi(0)+\Gamma_{0}^{t} S^{*}(T-t)\left(\lambda I+\Gamma_{0}^{T}\right)^{-1}\left(E h^{*}-S(T) \xi(0)\right) \\
& +\int_{0}^{t}\left[I-\Gamma_{s}^{t} S^{*}(T-t)\left(\lambda I+\Gamma_{s}^{T}\right)^{-1} S(T-t)\right] S(T-s) f\left(s, x_{s}^{\lambda}, u^{\lambda}(s)\right) d s \\
& +\int_{0}^{t}\left[I-\Gamma_{s}^{t} S^{*}(T-t)\left(\lambda I+\Gamma_{s}^{T}\right)^{-1} S(T-t)\right] S(T-s) g\left(s, x_{s}^{\lambda}, u^{\lambda}(s)\right) d w(s) \\
& +\int_{0}^{t} \Gamma_{s}^{t} S^{*}(T-t)\left(\lambda I+\Gamma_{s}^{T}\right)^{-1} \varphi(s) d w(s), \quad t \in J, \\
x^{\lambda}(t)= & \xi(t), \quad t \leq 0 .
\end{aligned}
$$

Letting $t=T$ in the equation above, it can be obtained that

$$
\begin{aligned}
x^{\lambda}(T)= & h^{*}-\lambda\left(\lambda I+\Gamma_{0}^{T}\right)^{-1}(E h-S(T) \xi(0)) \\
& -\lambda \int_{0}^{T}\left(\lambda I+\Gamma_{s}^{T}\right)^{-1} S(T-s) f\left(s, x_{s}^{\lambda}, u^{\lambda}(s)\right) d s \\
& -\lambda \int_{0}^{T}\left(\lambda I+\Gamma_{s}^{T}\right)^{-1}\left[S(T-s) g\left(s, x_{s}^{\lambda}, u^{\lambda}(s)\right)+\varphi(s)\right] d w(s) .
\end{aligned}
$$


Theorem 3.8 Under hypotheses $\left(\mathrm{A}_{1}\right),\left(\mathrm{A}_{2}^{*}\right)$, and $\left(\mathrm{A}_{3}\right)$, system $(1)$ is approximately controllable on $[0, T]$.

Proof By $\left(\mathrm{A}_{2}^{*}\right),\left|f\left(t, \eta_{1}, u_{1}\right)\right|^{p}+\left\|g\left(t, \eta_{1}, u_{1}\right)\right\|_{2}^{p} \leq K_{2}$ in $[0, T] \times \Omega$ for any $\eta_{1} \in B_{h}^{\alpha}$ and $u_{1} \in$ $U$. Then there exists a subsequence, still denoted by $\left\{f\left(s, x_{s}^{\lambda}, u^{\lambda}(s)\right), g\left(s, x_{s}^{\lambda}, u^{\lambda}(s)\right)\right\}$, weakly converging to some $\{f(s), g(s)\}$ in $H \times L_{2}^{0}(K, H)$. By $\left(\mathrm{A}_{3}\right)$, we also have $\left\|\lambda\left(\lambda I+\Gamma_{s}^{T}\right)^{-1}\right\| \leq 1$ for $0 \leq s \leq T$ [14]. From the compactness of $S(t)$, we obtain that, in $[0, T] \times \Omega$,

$$
\begin{aligned}
& S(T-s) f\left(s, x_{s}^{\lambda}, u^{\lambda}(s)\right) \rightarrow S(T-s) f(s), \\
& S(T-s) g\left(s, x_{s}^{\lambda}, u^{\lambda}(s)\right) \rightarrow S(T-s) g(s) .
\end{aligned}
$$

Meanwhile

$$
\begin{aligned}
E\left|x^{\lambda}(T)-h^{*}\right|^{p} & \\
\leq & 6^{p-1}\left|\lambda\left(\lambda I+\Gamma_{0}^{T}\right)^{-1}(E h-S(T) \xi(0))\right|^{p} \\
& +6^{p-1} E\left(\int_{0}^{T}\left\|\lambda\left(\lambda I+\Gamma_{0}^{T}\right)^{-1}\right\|\left|S(T-s)\left[f\left(s, x_{s}^{\lambda}, u^{\lambda}(s)\right)-f(s)\right]\right| d s\right)^{p} \\
& +6^{p-1} E\left(\int_{0}^{T}\left|\lambda\left(\lambda I+\Gamma_{0}^{T}\right)^{-1} S(T-s) f(s)\right| d s\right)^{p} \\
& +6^{p-1} E\left(\int_{0}^{T}\left\|\lambda\left(\lambda I+\Gamma_{0}^{T}\right)^{-1}\right\|^{2}\left\|S(T-s)\left[g\left(s, x_{s}^{\lambda}, u^{\lambda}(s)\right)-g(s)\right]\right\|_{2}^{2} d s\right)^{\frac{p}{2}} \\
& +6^{p-1} E\left(\int_{0}^{T}\left\|\lambda\left(\lambda I+\Gamma_{0}^{T}\right)^{-1} S(T-s) g(s)\right\|_{2}^{2} d s\right)^{\frac{p}{2}} \\
& +6^{p-1} E\left(\int_{0}^{T}\left\|\lambda\left(\lambda I+\Gamma_{0}^{T}\right)^{-1} \varphi(s)\right\|_{2}^{2} d s\right)^{\frac{p}{2}} .
\end{aligned}
$$

By the dominated convergence theorem and $\left(\mathrm{A}_{3}\right)$ (i.e., $\lambda\left(\lambda I+\Gamma_{s}^{T}\right)^{-1} \rightarrow 0$ in strong operator topology as $\left.\lambda \rightarrow 0^{+}\right), E\left|x^{\lambda}(T)-h^{*}\right|^{p} \rightarrow 0$ as $\lambda \rightarrow 0^{+}$, which implies the approximate controllability of system (1). The proof is completed.

Remark 3.9 It shall be noted that a similar but rather preliminary result has been developed in [23], where the initial datum of the system $\xi \in L^{p}\left(\Omega, C_{\alpha}^{h}\right)$ and the delays are considered in the space $L^{p}\left(\Omega, C_{\alpha}^{h}\right)$, where $C_{\alpha}^{h}=\left\{x \in C\left(R^{-}, H_{\alpha}\right) \mid \int_{-\infty}^{0} h(s) \sup _{s \leq \tau \leq 0}\|x(\tau)\|_{\alpha}^{p} d s<\infty\right\}$.

Since

$$
\begin{aligned}
\int_{-\infty}^{0} h(s) \sup _{s \leq \tau \leq 0}\|x(\tau)\|_{\alpha} d s & =\int_{-\infty}^{0} h(s)^{\frac{1}{q}} h(s)^{\frac{1}{p}} \sup _{s \leq \tau \leq 0}\|x(\tau)\|_{\alpha} d s \\
& \leq\left(\int_{-\infty}^{0} h(s) d s\right)^{\frac{1}{q}}\left(\int_{-\infty}^{0} h(s) \sup _{s \leq \tau \leq 0}\|x(\tau)\|_{\alpha}^{p} d s\right)^{\frac{1}{p}} \\
& =l^{\frac{1}{q}}\left(\int_{-\infty}^{0} h(s) \sup _{s \leq \tau \leq 0}\|x(\tau)\|_{\alpha}^{p} d s\right)^{\frac{1}{p}}
\end{aligned}
$$

it is clear that $B_{h}^{\alpha} \supset C_{\alpha}^{h}$. That is, a more general space is studied in this paper. Moreover, the case $0<\alpha<\frac{p-2}{2 p}$ is considered in our results, while the result in [23] needs $\alpha$ to be 
$0<\alpha<\frac{p-2}{4 p}$. Clearly, the result in [23] could not be well applied to the cases which are studied in this paper.

\section{An example}

Heat equation describes the flow of heat by conduction through a stationary homogeneous, isotropic material. As an application of our results, we consider a heat equation system described by the following stochastic partial differential equation:

$$
\left\{\begin{aligned}
d v(t, x)= & \left(-\frac{\partial^{2}}{\partial x^{2}} v(t, x)+b(x) u(t)+\int_{-\infty}^{t} e^{4(s-t)} v(s, x) d s\right) d t \\
& +\left(\int_{-\infty}^{t} e^{4(s-t)} v(s, x) d s+\log (1+|u(t)|)\right) d w(t), \\
& t \in J=[0, T], 0 \leq x \leq \pi \\
v(t, 0)= & v(t, \pi)=0, \quad t \geq 0, \\
v(s, x)= & \xi(s, x) \in L^{p}\left(\Omega, B_{h}^{\alpha}\right), \quad s \in(-\infty, 0], 0 \leq x \leq \pi .
\end{aligned}\right.
$$

Here $v(t, x)$ denotes the temperature at time $t . u(t)$ is the control term to enable the system temperature achieve the target value approximately for a given time $T$. Let $H=L^{2}(0, \pi)$ be endowed with the usual norm $\|\cdot\|_{L^{2}}, h(s)=e^{2 s}, \int_{\infty}^{0} h(s) d s=\frac{1}{2}$. Note that there exists a complete orthonormal set $\left\{e_{n}\right\}, n \geq 1$, of eigenvectors of $A$ with $e_{n}(x)=\sqrt{\frac{2}{\pi}}$. The analytic semigroup $S(t), t \geq 0$, is generated by $A$ such that

$$
\begin{aligned}
& A \rho=\sum_{n=1}^{\infty} n^{2}\left\langle\rho, e_{n}\right\rangle e_{n}, \quad \rho \in D(A), \\
& S(t) \rho=\sum_{n=1}^{\infty} e^{-n^{2} t}\left\langle\rho, e_{n}\right\rangle e_{n}, \quad \rho \in H .
\end{aligned}
$$

We define $A^{\alpha}$ (actually $|A|^{\alpha}$ ) for the self-adjoint operator $A$ by the classical spectral theorem, and it can be obtained that

$$
|A|^{\alpha} e^{-A t} \rho=\sum_{n=1}^{\infty}\left(n^{2}\right)^{\alpha} e^{-n^{2} t}\left\langle\rho, e_{n}\right\rangle e_{n},
$$

which yields

$$
\begin{aligned}
\left|A^{\alpha} e^{-A t} \rho\right|^{2} & =\sum_{n=1}^{\infty} n^{4 \alpha} e^{-2 n^{2} t}\left|\left\langle\rho, e_{n}\right\rangle\right|^{2} \\
& =\sum_{n=1}^{\infty}\left(n^{2} t\right)^{2 \alpha} e^{-\left(2 n^{2}-2 a\right) t}\left|\left\langle\rho, e_{n}\right\rangle\right|^{2} .
\end{aligned}
$$

On the other hand, for any $\zeta_{1}, \zeta_{2}$ and $\zeta \in H_{\alpha}$, we have

$$
\begin{aligned}
\|\zeta\|_{L^{2}}^{2} & =\int_{0}^{\pi}|\zeta(x)|^{2} d x \\
& =\sum_{n=1}^{\infty}\left\langle\zeta, e_{n}\right\rangle^{2}
\end{aligned}
$$




$$
\begin{aligned}
& \leq \sum_{n=1}^{\infty} n^{4 \alpha}\left\langle\zeta, e_{n}\right\rangle^{2} \\
& =\left|A^{\alpha} \zeta\right|^{2}=\|\zeta\|_{\alpha}^{2}
\end{aligned}
$$

and

$$
\begin{aligned}
\left\|\zeta_{2}-\zeta_{1}\right\|_{L^{2}} & =\int_{0}^{\pi}\left|\zeta_{2}(x)-\zeta_{1}(x)\right|^{2} d x \\
& =\sum_{n=1}^{\infty}\left\langle\zeta_{2}-\zeta_{1}, e_{n}\right\rangle^{2} \\
& \leq \sum_{n=1}^{\infty} n^{4 \alpha}\left\langle\zeta_{2}-\zeta_{1}, e_{n}\right\rangle^{2} \\
& =\left|A^{\alpha} \zeta\right|^{2}=\left\|\zeta_{2}-\zeta_{1}\right\|_{\alpha}^{2} .
\end{aligned}
$$

For any $\phi \in B_{h}, \phi(s)(x)=\phi(s, x),(s, x) \in(-\infty, 0] \times[0, \pi]$, it is clear that $f(t, \phi)=g(t, \phi)=$ $\int_{-\infty}^{0} e^{4 s} \phi(s)(x) d s$, and for $\phi, \psi \in B_{h}$,

$$
\begin{aligned}
\|f(t, \phi)-f(t, \psi)\|_{L^{2}} & =\left[\int_{0}^{\pi}\left(\int_{-\infty}^{0} e^{4 s}(\phi(s)(x)-\psi(s)(x)) d s\right)^{2} d x\right]^{\frac{1}{2}} \\
& \leq\left(\int_{-\infty}^{0} e^{4 s} d s\right)^{\frac{1}{2}}\left(\int_{0}^{\pi} \int_{-\infty}^{0} e^{4 s}(\phi(s)(x)-\psi(s)(x))^{2} d s d x\right)^{\frac{1}{2}} \\
& =\frac{1}{2}\left(\int_{-\infty}^{0} e^{4 s} \int_{0}^{\pi}(\phi(s)(x)-\psi(s)(x))^{2} d x d s\right)^{\frac{1}{2}} \\
& =\frac{1}{2}\left(\int_{-\infty}^{0} e^{4 s}\|\phi(s)-\psi(s)\|_{L^{2}}^{2}\right)^{\frac{1}{2}} \\
& \leq \frac{1}{2}\left(\int_{-\infty}^{0} e^{4 s}\left[\sup _{s \leq \tau \leq 0}\|\phi(s)-\psi(s)\|_{L^{2}}\right]^{2}\right)^{\frac{1}{2}} \\
& \leq \int_{-\infty}^{0} e^{2 s} \sup _{s \leq \tau \leq 0}\|\phi(s)-\psi(s)\|_{L^{2}} d s \\
& =\frac{1}{2}\|\phi-\psi\|_{B_{h}^{\alpha} .}
\end{aligned}
$$

Then the functions $f(t, \phi)$ and $g(t, \phi)$ are globally Lipschitz continuous in $\phi \in B_{h}^{\alpha}$ and uniformly bounded. On the other hand, it is known that the deterministic linear system corresponding to (4) is approximately controllable on every $[0, t], t>0$, provided that for $n=1,2, \ldots$,

$$
\int_{0}^{\pi} b(x) e_{n}(x) d x \neq 0
$$

By Theorem 3.8, we can ensure that system (4) is approximately controllable on $[0, T]$.

Remark 4.1 In system (4), considering the initial datum $\xi$, if let $\|\xi(s)\|_{\alpha}=e^{-\frac{3}{2} s}$, for $\infty<$ $s \leq 0$, it can be seen that the result in [23] cannot be applied. Obviously, our results include such intractable cases. 


\section{Conclusion}

In this paper, improved approximate controllability results of a class of stochastic partial differential systems with infinite delays are obtained in a general case by using a fixed point theorem, stochastic analysis techniques and an important lemma established, which fills a gap of the research area of control theory for stochastic functional partial differential systems. Moreover, intuitively, under more hypothesis, the controllability results established in this study could be also extended to the case of neutral type, which are frequently used to characterize some Burgers equations, vibration equations, Navier-Stokes equations, etc. [30]. Thus, it is an important and interesting topic to further study controllability problems of the neutral stochastic partial differential systems, and the problems will be focused on in our future studies.

\section{Competing interests}

The authors declare that they have no competing interests.

\section{Authors' contributions}

All authors contributed equally in writing this article. All authors read and approved the final manuscript.

\section{Author details}

${ }^{1}$ School of Statistics and Mathematics, Zhongnan University of Economics and Law, Wuhan, 430073, P.R. China. ${ }^{2}$ Key Laboratory of Advanced Technology for Materials Synthesis and Processing, Wuhan University of Technology, Wuhan, P.R. China.

\section{Acknowledgements}

The authors sincerely thank the reviewers for their valuable comments and the editor for kind help on this paper. This research was supported by the National Natural Science Foundation of China (Grant No. 11301544).

Received: 3 November 2014 Accepted: 3 March 2015 Published online: 10 March 2015

\section{References}

1. Caraballo, T: Nonlinear partial functional differential equations: existence and stability. J. Math. Anal. Appl. 262, 87-111 (2001)

2. Muller, TG, Timmera, J: Fitting parameters in partial differential equations from partially observed noisy data. Physica D 171, 1-7 (2002)

3. Kevrekidis, PG: On a class of discretizations of Hamiltonian nonlinear partial differential equations. Physica D 183, 68-86 (2003)

4. Pazy, A: Semigroups of Linear Operators and Applications to Partial Differential Equations. Springer, Berlin (1983)

5. Friedman, A: Stochastic Differential Equations and Applications. Academic Press, New York (1975)

6. Mao, X: Stochastic Differential Equations and Applications. Ellis Horwood, Chichester (1997)

7. Ockendon, J, Howison, S, Lacey, A, Movchan, A: Applied Partial Differential Equations. Oxford University Press, New York (1999)

8. Taniguchi, T: Successive approximations to solutions of stochastic differential equations. J. Differ. Equ. 96, 152-169 (1992)

9. Taniguchi, T, Liu, K, Truman, A: Existence, uniqueness and asymptotic behavior of mild solutions to stochastic functional differential equations in Hilbert spaces. J. Differ. Equ. 81, 72-91 (2002)

10. Da Prato, G, Zabczyk, J: Stochastic Equations in Infinite Dimensions. Cambridge University Press, Cambridge (1992)

11. Mahmudov, NI, Denker, A: On controllability of linear stochastic systems. Int. J. Control 73, 144-151 (2000)

12. Mahmudov, NI: Controllability of linear stochastic systems in Hilbert spaces. J. Math. Anal. Appl. 259, 64-82 (2001)

13. Mahmudov, NI: Controllability of linear stochastic systems. IEEE Trans. Autom. Control 41, 724-731 (2001)

14. Bashirov, AE, Mahmudov, NI: On concepts of controllability for deterministic and stochastic systems. SIAM J. Control Optim. 37, 1808-1821 (1999)

15. Mahmudov, NI: Controllability of semilinear stochastic systems in Hilbert spaces. J. Math. Anal. Appl. 288, 197-211 (2003)

16. Dauer, JP, Mahmudov, NI: Controllability of stochastic semilinear functional differential equations in Hilbert spaces. J. Math. Anal. Appl. 290, 373-394 (2004)

17. Balachandran, K, Sakthivel, R: Controllability of neutral functional integrodifferential systems in Banach spaces. Comput. Math. Appl. 39, 117-126 (2000)

18. Balachandran, $\mathrm{K}, \mathrm{Kim}, \mathrm{JH}$, Karthikeyan, S: Controllability of semilinear stochastic integrodifferential equations. Kybernetika 43, 31-44 (2007)

19. Hale, JK, Kato, J: Phase space for retarded equations with infinite delay. Funkc. Ekvacioj 21, 11-41 (1978)

20. Hale, JK: Theory of Functional Differential Equations. Springer, Berlin (1977)

21. Balasubramaniam, P, Ntouyas, SK: Controllability for neutral stochastic functional differential inclusions with infinite delay in abstract space. J. Math. Anal. Appl. 324, 161-176 (2006)

22. Balachandran, K, Anandhi, ER: Controllability of neutral functional integrodifferential infinite delay systems in Banach spaces. Taiwan. J. Math. 8, 689-702 (2004) 
23. Bao, $\mathrm{H}$, Jiang, D: The Banach spaces and with application to the approximate controllability of stochastic partial functional differential equations with infinite delay. Stoch. Anal. Appl. 25, 995-1024 (2007)

24. Liu, B: Controllability of neutral functional differential and integrodifferential inclusions with infinite delay. J. Optim. Theory Appl. 123, 573-593 (2004)

25. Liu, B: Controllability of nonlinear neutral evolution integrodifferential systems with infinite delay. J. Optim. Theory Appl. 122, 87-109 (2004)

26. Caraballo, T, Langa, JA, Taniguchi, T: The exponential behaviour and stabilizability of stochastic 2D-Navier-Stokes equations. J. Differ. Equ. 179, 714-737 (2002)

27. Temam, R: Infinite Dimensional Dynamical Systems in Mechanics and Physics. Springer, Berlin (1988)

28. Palazoglu, A, Karakas, A: Control of nonlinear distributed parameter systems using generalized invariants. Automatica 179, 714-734 (2002)

29. Carmona, RA, Rozovskii, B: Stochastic Partial Differential Equations: Six Perspectives. Am. Math. Soc., New York (1998)

30. Adomian, G: Nonlinear Stochastic Systems Theory and Applications to Physics. Springer, Berlin (2001)

31. Moran, MJ, Shapiro, HN: Fundamentals of Engineering Thermodynamics. Wiley, New York (1988)

32. Balachandran, K, Dauer, JP: Null controllability of nonlinear infinite delay systems with time varying multiple delays in control. Appl. Math. Lett. 9, 115-121 (1996)

33. Dauer, JP, Balachandran, K, Anthoni, SM: Null controllability of nonlinear infinite neutral systems with delays in control. Appl. Math. Lett. 36, 39-50 (1998)

\section{Submit your manuscript to a SpringerOpen ${ }^{\mathcal{O}}$ journal and benefit from:}

- Convenient online submission

- Rigorous peer review

Immediate publication on acceptance

- Open access: articles freely available online

- High visibility within the field

- Retaining the copyright to your article 